\title{
Behavioral Status Influences the Dependence of Odorant- Induced Change in Firing on Prestimulus Firing Rate
}

\author{
(1DAnan Li, ${ }^{1,5 *}$ E. Mae Guthman, ${ }^{3,4 *}$ Wilder T. Doucette, ${ }^{6}$ and Diego Restrepo ${ }^{1,2,3}$ \\ ${ }^{1}$ Department of Cell and Developmental Biology, ${ }^{2}$ Rocky Mountain Taste and Smell Center, ${ }^{3}$ Neuroscience Program, and ${ }^{4}$ Department of Pharmaceutical \\ Sciences, University of Colorado Anschutz Medical Campus, Aurora, Colorado 80045, ${ }^{5}$ Jiangsu Key Laboratory of Brain Disease and Bioinformation, \\ Research Center for Biochemistry and Molecular Biology, Xuzhou Medical University, Xuzhou, 221004, China, and ${ }^{6}$ Department of Psychiatry, Geisel School \\ of Medicine at Dartmouth College, Hanover, New Hampshire 03755
}

The firing rate of the mitral/tufted cells in the olfactory bulb is known to undergo significant trial-to-trial variability and is affected by anesthesia. Here we ask whether odorant-elicited changes in firing rate depend on the rate before application of the stimulus in the awake and anesthetized mouse. We find that prestimulus firing rate varies widely on a trial-to-trial basis and that the stimulus-induced change in firing rate decreases with increasing prestimulus firing rate. Interestingly, this prestimulus firing rate dependence was different when the behavioral task did not involve detecting the valence of the stimulus. Finally, when the animal was learning to associate the odor with reward, the prestimulus firing rate was smaller for false alarms compared with correct rejections, suggesting that intrinsic activity reflects the anticipatory status of the animal. Thus, in this sensory modality, changes in behavioral status alter the intrinsic prestimulus activity, leading to a change in the responsiveness of the second-order neurons. We speculate that this trial-to-trial variability in odorant responses reflects sampling of the massive parallel input by subsets of mitral cells.

Key words: anticipatory; associative learning; olfaction; reward; sensory; top-down

\section{Significance Statement}

The olfactory bulb must deal with processing massive parallel input from $\sim 1200$ distinct olfactory receptors. In contrast, the visual system receives input from a small number of photoreceptors and achieves recognition of complex stimuli by allocating processing for distinct spatial locations to different brain areas. Here we find that the change in firing rate elicited by the odorant in second-order mitral cells depends on the intrinsic activity leading to a change of magnitude in the responsiveness of these neurons relative to this prestimulus activity. Further, we find that prestimulus firing rate is influenced by behavioral status. This suggests that there is top-down modulation allowing downstream brain processing areas to perform dynamic readout of olfactory information.

\section{Introduction}

The brain must perceive the ever-changing external context accurately to determine appropriate responses to sensory stimuli.

\footnotetext{
Received Oct. 8, 2016; revised Jan. 7, 2017; accepted Jan. 8, 2017.

Author contributions: A.L., W.T.D., and D.R. designed research; A.L. and W.T.D. performed research; A.L., E.M.G., and D.R. analyzed data; A.L., E.M.G., W.T.D., and D.R. wrote the paper.

This work was supported by National Institutes of Health/National Institute on Deafness and Other Communication Disorders Grants R01 DC00566, F32 DC011980, and P30 DC04657, and National Natural Science Foundation of China Grant NSFC 31571082. We thank Nicole Arevalo, Jamie Costabile, Ling Gong, and Qing Liu for technical assistance; we thank Douglas Curran-Everett for assistance with statistics; and we thank Gidon Felsen, Alexia Nunez-Parra, Ming Ma, Baris Ozbay, Daniel Ramirez-Gordillo, Shane Rolen, Nathan Schoppa, Elizabeth A. Stubblefield, and John Thompson for comments on the manuscript and data analysis.

The authors declare no competing financial interests.

${ }^{*}$ A.L. and E.M.G. contributed equally to this study.

Correspondence should be addressed to Dr. Diego Restrepo, University of Colorado Anschutz Medical Campus, Department of Cell and Developmental Biology, 12801 East 17th Avenue, Bldg RC1 South, Room L18-11119, Aurora,C0 80045. E-mail: diego.restrepo@ucdenver.edu.
}

In the olfactory system, this process is particularly complex because of the need to process parallel input from $\sim 1200$ olfactory receptors (Buck and Axel, 1991; Touhara and Vosshall, 2009). Further complicating the issue, the olfactory bulb (OB) receives massive top-down modulation of sensory processing that depends on a variety of factors, such as attention, motivation, and arousal (Eeckman and Freeman, 1991; Kay and Freeman, 1998; Fontanini and Katz, 2008; Poulet and Petersen, 2008; Zhang et al., 2014; Otazu et al., 2015). Thus, the activity of mitral/tufted (M/T) cells, second-order neurons of the $\mathrm{OB}$, is modulated by considerable feedforward and feedback input.

Interestingly, differences in the neuronal activity of $\mathrm{M} / \mathrm{T}$ cells have been observed across varying brain states. In fundamental studies, Lord Adrian found increased irregular intrinsic activity 
A

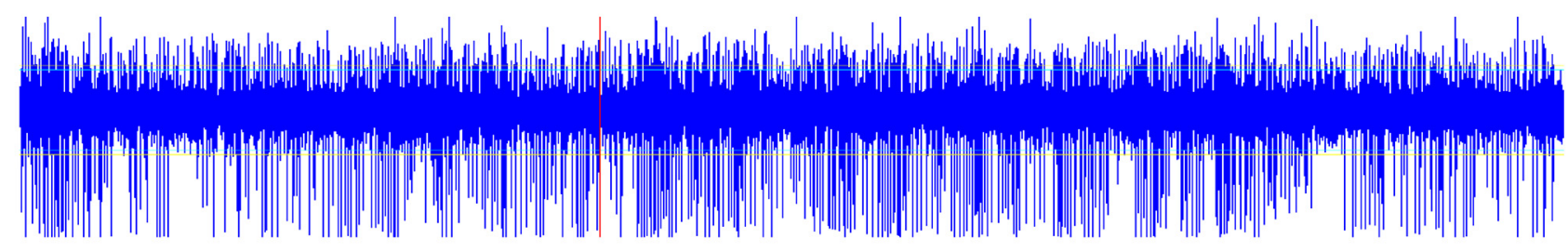

Odor / $2.5 \mathrm{~s}$

B

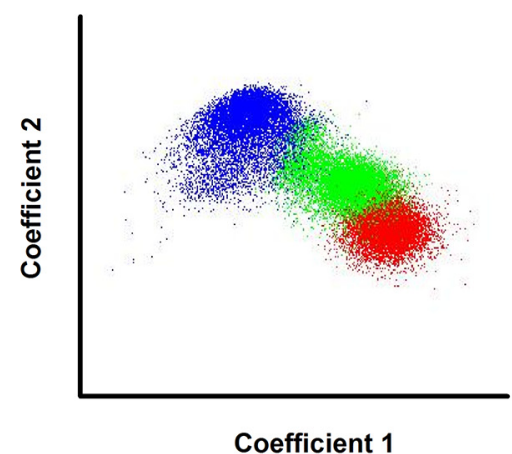

C

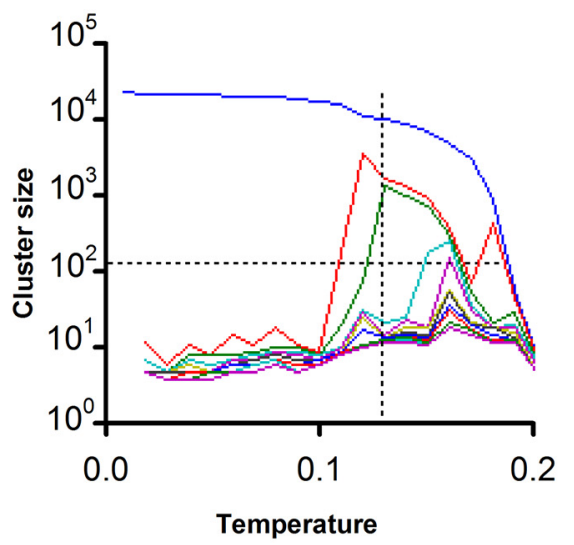

F-1

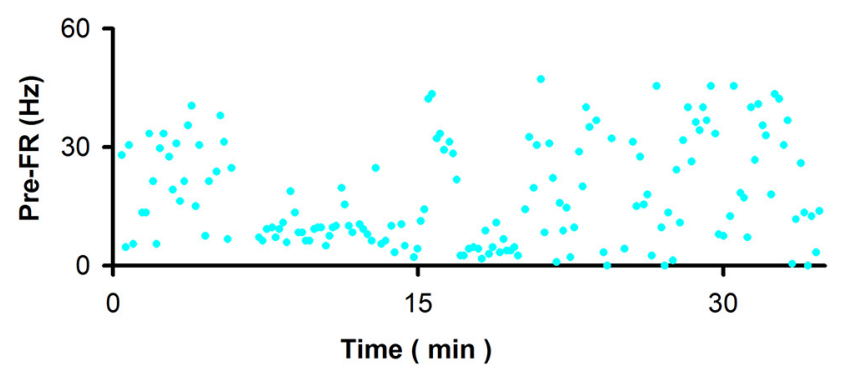

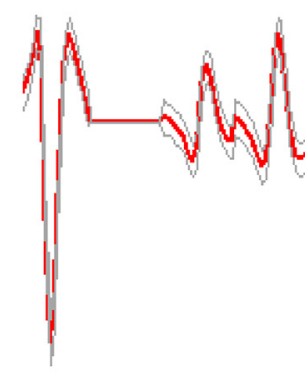

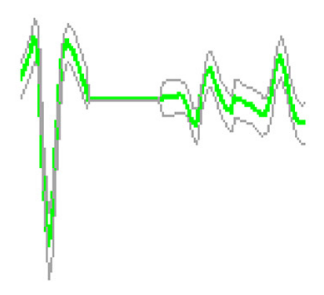

E

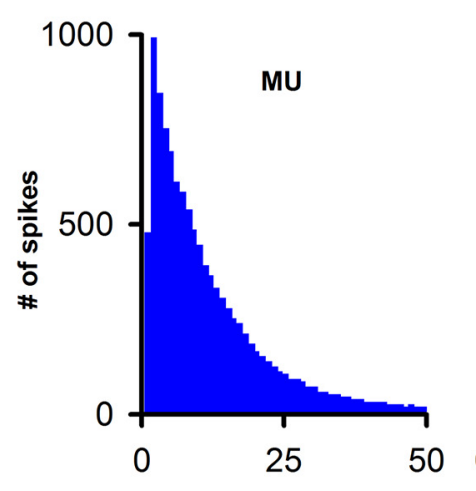

F-2
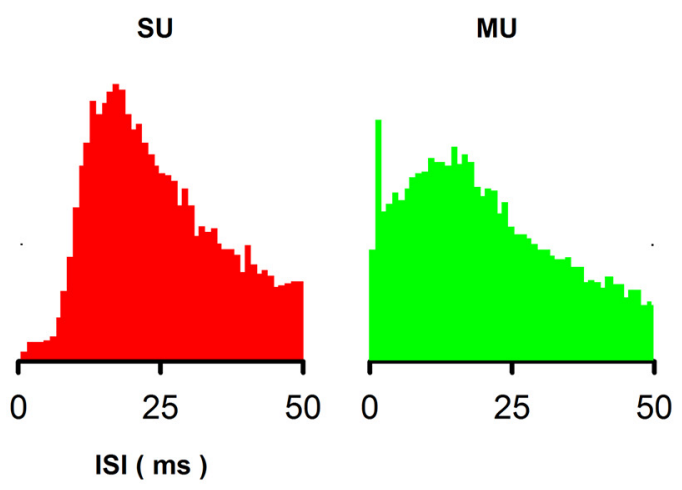

50

Figure 1. Tetrode data acquisition during awake behaving recording. $\boldsymbol{A}$, Example of raw data (spikes, $300-5000 \mathrm{~Hz}$ ) recorded during $9 \mathrm{~s}$ for one trial (sample rate $24 \mathrm{kHz}$ ). Red vertical line indicates onset of odorant stimulation. $\boldsymbol{B}-\boldsymbol{D}$, Example of spike detection and sorting using wavelet analysis with superparamagnetic clustering of extracellular voltage recorded in tetrodes resulting in separation of 1 SU and 2 MUs (Li et al., 2014, 2015). B, Separation of tetrode spikes shown under $\boldsymbol{C}$ into three different units. The two coefficients were wavelets for spike shape in two of the electrodes in the tetrode. D, Temperature chosen under paramagnetic clustering to obtain three different units (the meaning of temperature under paramagnetic clustering is explained by Li et al., 2014, 2015). $\boldsymbol{E}$, ISI histograms for the three units. Two are MUs and one is an SU.F, Example of the stability of the spike waveform throughout a go-no go session. $\boldsymbol{F 1}$, Trial-by-trial variability for pre-FR for this SU. F2, Spike waveforms remain stable throughout the session. Solid line indicates mean field potential. Light lines indicate mean \pm SD ( $n=30$ trials). Bottom, First 30 trials. Top, Last 30 trials in the session. $\boldsymbol{D}, \mathbf{F 2}$, The spike width is $1 \mathrm{~ms}$. 
A-1

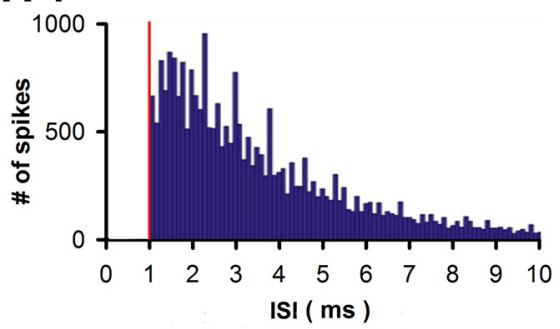

A-4

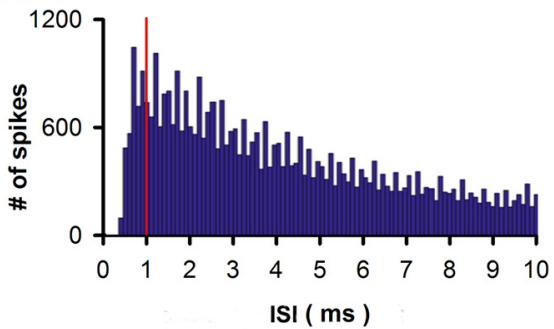

ISI ( ms )
A-2

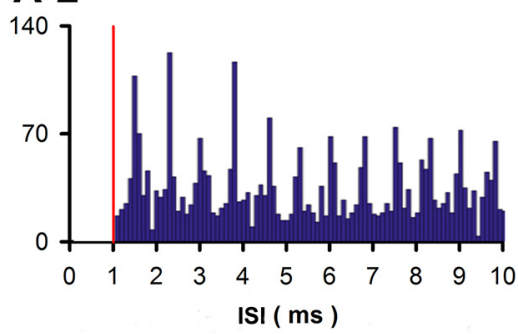

A-5

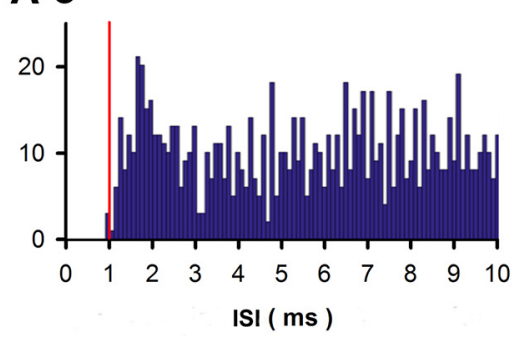

B-2
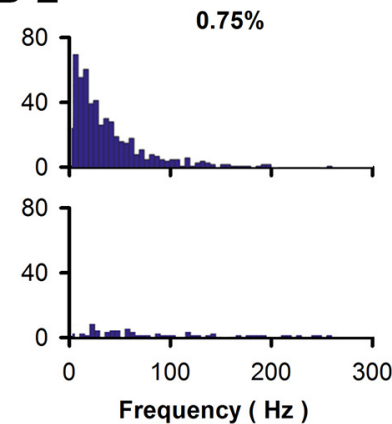

C-2
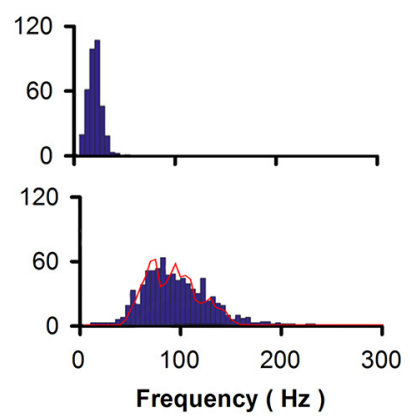

D-2

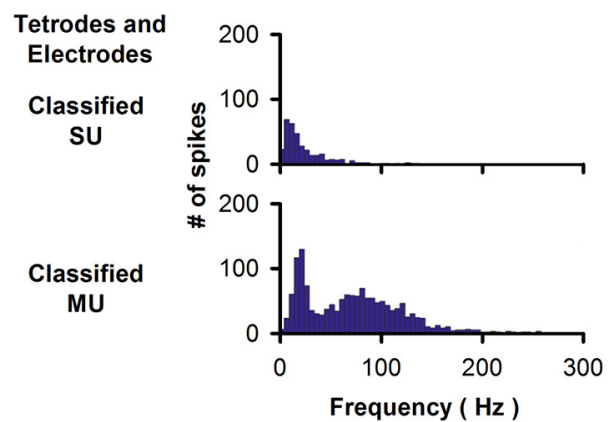

A-3

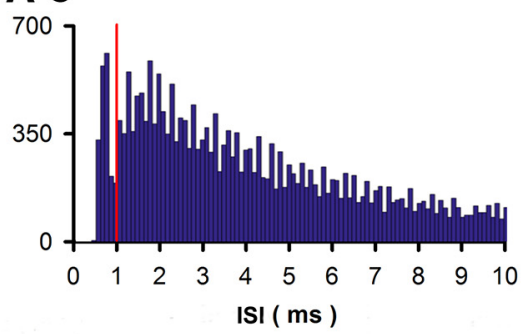

A-6

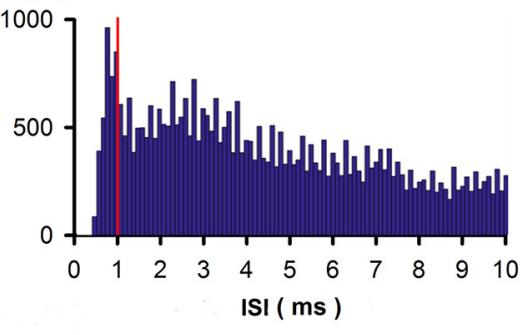

B-3
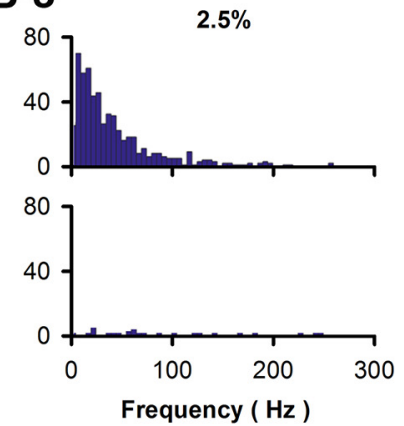

C-3

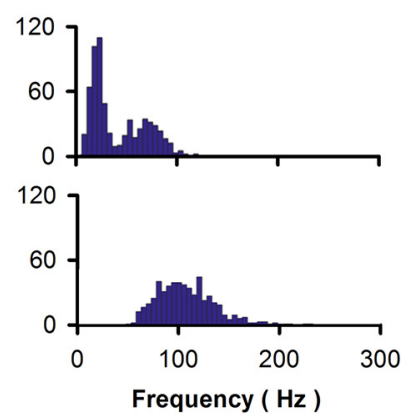

D-3

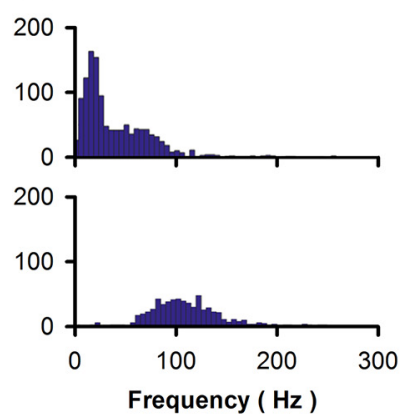

Figure 2. Classification of units to SUs based on the criterion that $<0.75 \%$ of the ISIs are $<1$ ms results in unimodal pre-FR distributions regardless of whether recordings were with multielectrode arrays or tetrodes. A1-A6, Examples of six ISIs distributions for spikes recorded in entire sessions. A1-A3, Units for ISIs were recorded with tetrodes. A4-A6, Units for ISIs were recorded with multielectrode arrays. Vertical red lines are placed at $1 \mathrm{~ms}$. $\boldsymbol{B}-\boldsymbol{D}$, Pre-FR histograms for units classified as single or MUs using different percent of (Figure legend continues.) 
in the $\mathrm{OB}$ as the animal awoke from anesthesia (Adrian, 1950). Intriguingly, he observed that this increase in intrinsic (prestimulus) activity made it difficult to detect odorant responses in $\mathrm{M} / \mathrm{T}$ cells and hypothesized that this effect could be altered by changes in attention. Recent studies have confirmed that, in animals under anesthesia, $\mathrm{M} / \mathrm{T}$ cells responded to odorants with robust increases in firing rate (FR), whereas they display irregular prestimulus activity and weak responses in awake animals (Rinberg et al., 2006; Gire et al., 2013; Gschwend et al., 2015).

Weak responses in awake animals can be partially explained by the fact that odorant responses take place at a specific phase within the sniff cycle (Cury and Uchida, 2010; Shusterman et al., 2011; Wachowiak, 2011; Blauvelt et al., 2013; Li et al., 2015). However, recent studies revealed "silent" M/T cells with low prestimulus FR (pre-FR) and postulated that these cells dominate the excitatory odorant response in awake mice (Kollo et al., 2014). In addition, studies in OB slices indicated that individual $\mathrm{M} / \mathrm{T}$ cells switch between states with low and high pre-FR (Heyward et al., 2001; Roux et al., 2015) and exhibit glomerulus-wide long-lasting depolarizations (Carlson et al., 2000). Further, recent work examining olfactory responses in Caenorhabditis elegans demonstrated bistability in the activity of second-order olfactory neurons that predicted changes in downstream neuron responses and the animal's behavior in response to an odorant (Gordus et al., 2015). These data raise the question whether individual M/T cells switch from low to high pre-FR in the awake animal with behavioral consequences.

In the present study, we found that individual $\mathrm{M} / \mathrm{T}$ cells show trial-to-trial variability in pre-FR that predicts both neuronal and behavioral responses to odorants.

\section{Materials and Methods}

Experiments. The analysis presented in this manuscript was performed with data obtained in the experiments described in previous publications (Doucette and Restrepo, 2008; Doucette et al., 2011; Li et al., 2014, 2015). The passive task data was obtained in the go-go passive odorant exposure experiment of Li et al. (2015) where two odorants were applied and the mouse was rewarded in $70 \%$ of the trials regardless of the odor. The optogenetic olfactory sensory neuron activation data were from the tetrode recording experiment in freestanding mice described by Li et al. (2014). Finally, the active task data was from the associative learning go-no go experiments (Doucette and Restrepo, 2008; Doucette et al., 2011; Li et al., 2015). In these experiments, the mouse was rewarded for licking for the rewarded $\left(\mathrm{S}^{+}\right)$and not for the unrewarded $\left(\mathrm{S}^{-}\right)$odorant. Thus, the analysis of the data performed in those publications is directly related to this publication. Below we describe the experiments and refer to these publications on data analysis that is pertinent to the current publication.

Animals. Both C57BL/6J (RRID:IMSR_JAX:000664) (Doucette and Restrepo, 2008; Doucette et al., 2011; Li et al., 2014, 2015) and OMP-

\section{$\leftarrow$}

(Figure legend continued.) $\quad \mid \mathrm{Sls}<1 \mathrm{~ms}$. B, Pre-FR histograms for units recorded using tetrodes (595 units) (Li et al., 2015). C, pre-FR histograms for units recorded using multielectrode arrays (1163 units) (Doucette and Restrepo, 2008). D, pre-FR histograms for units recorded using either tetrodes or multielectrode arrays (1758 units) (data from both Doucette and Restrepo, 2008 and Li et al., 2015). SUs were classified based on the following percent of ISIs falling within 0-1 ms: $0 \%(B 1, C 1, D 1), 0.75 \%(B 2, C 2, D 2)$, or $2.5 \%(B 3, C 3, D 3)$. Using $0 \%$ results in a bimodal pre-FR distribution for units classified as MUs, whereas $2.5 \%$ results in a bimodal pre-FR distribution for units classified as SUs. As expected for single sharp electrodes (Gray et al., 1995), multielectrode array recordings include a large fraction of MUs. (2, Red line indicates information on the number of SUs making up each MU in the pre-FR distribution. This line was generated by fitting the MU pre-FR with the SU pre-FR with varying fractions of MUs made up by different numbers of SUs. The best fit was with the following fractions of MUs made up by different numbers of SUs: 0.12 for 2 units, $0.29 / 3,0.23 / 4,0.2 / 5,0.11 / 6,0.06 / 7$.
hChR2V (RRID:IMSR_JAX:014173) (Li et al., 2014) male mice (mus musculus) were used in experiments analyzed in this study (2-8 months old). The OMP- $h C h R 2 V$ mice were produced by gene targeting as described in our previous study (Li et al., 2014). All the mice were bred in the animal facilities of the University of Colorado Anschutz Medical Campus. We used male adult heterozygous OMP-ChR2 mice and wildtype C57BL/6J mice. The animals were housed in a vivarium with a 14:10 h light-dark cycle with lights on at 10:00 P.M. Food (Teklad Global Rodent Diet no. 2918, Harlan) was available ad libitum. Access to water was restricted to the behavioral session; however, if mice did not obtain $\sim 1 \mathrm{ml}$ of water during the behavioral session, additional water was provided in a dish in the cage (Li et al., 2015). All mice were weighed daily and received sufficient water during behavioral sessions to maintain $>80 \%$ of prewater restriction weight. All experiments were performed according to protocols approved by the University of Colorado Anschutz Medical Campus Institutional Animal Care and Use Committee.

Surgery for implantation of multielectrode arrays, tetrodes, and optotetrodes. Mice were briefly exposed to isoflurane (2.5\%); and subsequently, anesthesia was maintained by intraperitoneal ketamine-xylazine injection $(100 \mu \mathrm{g} / \mathrm{g}$ and $20 \mu \mathrm{g} / \mathrm{g}$, respectively) verified by toe pinch. On a stereotaxic device, the fur on the surface of the scalp from the midline of the orbits to the midpoint between the ears was removed, and a hole was drilled above the left $\mathrm{OB}$ for tetrodes or optotetrode implant $(4.0 \mathrm{~mm}$ anterior from bregma, $0.5 \mathrm{~mm}$ lateral from midline), and two screw holes were drilled into the parietal; one served as ground (Li et al., 2014, 2015).

The optotetrodes included one glass tube for the optic fiber and four tetrodes that consisted of four polyimide-coated nichrome wires (diameter $12.5 \mu \mathrm{m}$; Sandvik) gold plated to an impedance of $0.2-0.4 \mathrm{M} \Omega$ at $1 \mathrm{kHz}$. Tetrodes were connected, and the glass tube was glued through an EIB-16 interface board (Neuralynx). Multielectrode arrays, manufactured by Micro Probe, were constructed of platinum iridium wire etched to a $2 \mu \mathrm{m}$ tip and coated with parylene $\mathrm{C}$ to an impedance between 3 and $4 \mathrm{M} \Omega$ at $1 \mathrm{kHz}$. The electrodes/tetrodes/optotetrodes were implanted targeting the mitral cell layer in the $\mathrm{OB}$ determined by M/T firing (Doucette and Restrepo, 2008; Li et al., 2014) and sealed to the bone by dental acrylic.

Active associative learning (go-no go) and passive odorant exposure (gogo) tasks. We used instrumental conditioning in freely moving mice in an olfactometer (Doucette and Restrepo, 2008; Li et al., 2014). Briefly, for the go-no go task, the water-deprived mice started the trial by deciding to place their head in a sampling chamber for a preafferent period (Kay and Freeman, 1998) that varied randomly between 1 and $1.5 \mathrm{~s}$ before stimulation with odor. At this point, odorant stimulation started and the animals were required to stay at the port at least $0.5 \mathrm{~s}$ after odor onset. Mice obtained water delivery for the reinforced odor when they licked at least once in four $0.5 \mathrm{~s}$ segments $0.5-2.5 \mathrm{~s}$ after the onset of the odor. There was no penalty for licking before odor delivery. The odor port is located above the lick tube, in front of the animal's nose, and odor delivery takes place in $<300 \mathrm{~ms}$. When exposed to the unreinforced odorant, they quit licking because of the substantial effort required for licking the tube. All mice were first trained to distinguish $10 \%$ isoamyl acetate $(\mathrm{v} / \mathrm{v})($ reinforced odorant, $\mathrm{S}^{+}$) versus mineral oil (unreinforced odorant, $\mathrm{S}^{-}$). The animal's performance was evaluated in blocks of 20 trials ( 10 reinforced and 10 unreinforced presented at random), and each session included 6-10 blocks. Each block's percent correct value represents the percent of trials in which the odors were correctly discriminated and associated with the appropriate behavioral action. Once the animal learned to discriminate between isoamyl acetate and mineral oil in 1-3 sessions, they were ready for the go-no go novel odorant discrimination task. For novel odorant discrimination in go-no go experiments, we used odors that we found in earlier work stimulate ventral M/Ts (Doucette and Restrepo, 2008; Li et al., 2015). Finally, in go-go experiments, the animal obtained water reward in $70 \%$ of trials if they licked for $2 \mathrm{~s}$ during exposure to either of the two odors. The odorants used as $\mathrm{S}^{+} / \mathrm{S}^{-}$odors in the go-no go experiments and as one of the two odors in the go-go experiments are detailed previously (Doucette and Restrepo, 2008; Li et al., 2015).

In the go-no go experiment, performance and unit activity were evaluated for rewarded or unrewarded odorants in time periods when the animal was learning valence of the stimulus ("learning" segment defined 

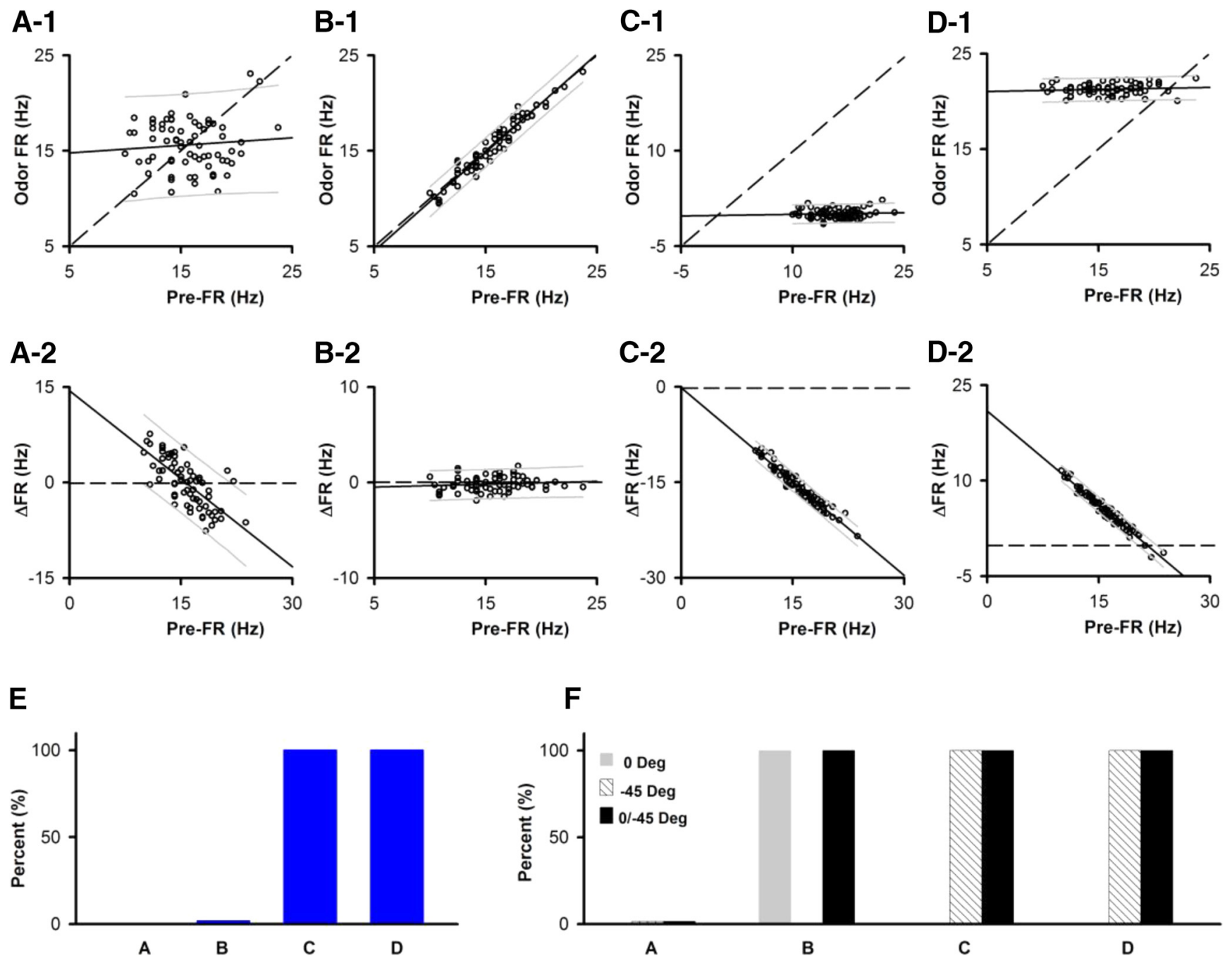

Figure 3. Statistical test of linearity and difference in the slope from 1 for simulation of different dependences of odor FR on pre-FR. The pre-FR dependence for 1451 SU-odor pairs recorded from in the go-no go task (examples shown in Fig. 4D1-F1) were resampled to yield different odor FR versus pre-FR dependences. $\boldsymbol{A}-\boldsymbol{D}$, Examples of odor $F R$ versus pre-FR $(\boldsymbol{A 1}, \boldsymbol{B} 1, \mathbf{C 1}, \mathbf{D 1})$ or $\Delta F R$ versus pre-FR $(\mathbf{A 2}, \mathbf{B 2}, \mathbf{C 2}, \mathbf{D 2})$ for the different dependences generated by resampling the pre-FR for the SU shown in Figure 4F1. Solid line indicates a best fit of the data. Dashed line indicates odor FR $=$ pre-FR or $\triangle F R=0$. Thin lines above and below the solid line indicate 5 th and 95 th percentile estimates. $A, 0$ dor $F R$ and pre-FR are independently resampled from the pre-FR distribution. $B, 0$ dor $F R=$ pre-FR + random normal noise with an SD equal to one-fourth of the $S D$ of the pre-FR distribution. $C, 0$ dor $F R=0+$ random normal noise with a SD equal to one-fourth of the $S D$ of the pre-FR distribution. $\boldsymbol{D}, 0$ dor $F R=95$ percentile pre-FR + random normal noise with a SD equal to one-fourth of the SD of the pre-FR distribution. Best-fit slope and intercept (Hz): $\boldsymbol{A 1}, 0.08,14.4 ; \boldsymbol{A 2},-0.92$, $14.3 ; \boldsymbol{B} 1,1.03,-0.62 ; \boldsymbol{B 2}, 0.03,-0.62 ; \mathbf{C}, 0.018,-0.17 ; \mathbf{C},-0.98,-0.17 ; \mathbf{D 1}, 0.02,20.9 ; \mathbf{D 2},-0.98,20.9 . \boldsymbol{E}, \boldsymbol{F}$, The dependences shown in $\boldsymbol{A}-\boldsymbol{E}$ were simulated for the 1451 SU- 0 dorant pairs recorded in the go-no go experiments (Fig. 4) and were then tested for difference of the slope from $1(\boldsymbol{E})$ or linearity $(\boldsymbol{F})$ for odor FR versus pre-FR with correction for multiple comparisons as explained in Materials and Methods. E, Percent of slopes different from 1.F, Percent linear. Gray represents percent significant for an $F$ test for linear best fit of odor FR versus pre-FR ( 0 degrees). Dashed lines indicate $F$ test for linear best fit of odor FR versus pre-FR performed after the axes were rotated by -45 degrees. Black represents percent significant in either the 0 or -45 degrees tests.

as when the animal was performing $\geq 45 \%$ and $\leq 65 \%$ correct trials) or in the period when the animal had learned to respond to the rewarded odorant ("retrieval" segment, defined as when the animal was performing $\geq 80 \%$ correct trials).

Odorant delivery. Odorant delivery was measured using a photoionization detector (mini-PID; Aurora Scientific) (Li et al., 2014, 2015). Odorant delivery occurred $0.3 \mathrm{~s}$ after turning the valve on and the timing was corrected to 0 at the time for odorant delivery.

Recording setup. The recording setup is as described previously (Doucette and Restrepo, 2008; Doucette et al., 2011; Li et al., 2014, 2015). The output of the electrodes/tetrodes was directed to a TDT $1 \times$ gain headstage that was in turn connected to either a CWE 16-channel amplifier or an A-M Systems 3600 amplifier. The signal was amplified 1000 times before digitizing with a Data Translation DT3010 A/D card in a PC. Data were acquired at $24 \mathrm{kHz}$ with custom software written in MATLAB (The MathWorks, RRID:SCR_001622).

During optogenetic light stimulation recording, the mouse was freely moving in a chamber $(11.6 \mathrm{~cm} \times 9.7 \mathrm{~cm} \times 9.4 \mathrm{~cm}$ deep $)$. Light was delivered via a diode-pumped, solid-state laser (473 nm; Shanghai Laser and Optics Century). The power of the laser was measured at the laser source and was found to be $19.5-21.5 \mathrm{~mW}$ for pulses 5-200 ms. Light stimulation was triggered by the recording program, which sent a signal to a stimulator (Master 8, A.M.P.I.). Pulse duration was $200 \mathrm{~ms}$. Light pulses were presented for 60 trials with an intertrial interval of $20 \mathrm{~s}$.

Monitoring pressure change during sniffing. Sniffing was monitored by recording intranasal pressure through an implanted nasal cannula connected to a pressure sensor (Model 24PCEFA6G(EA), 0-0.5 psi, Honeywell) via polyethylene tubing. Surgical procedures for cannula implantation were performed as described previously (Li et al., 2014, 2015). Voltage recording of pressure transients was amplified 100-fold using a 3000 amplifier from A-M Systems, digitized using a DT3010 A/D card from Data Translation in a PC, and sampled at $24 \mathrm{kHz}$. Each sniff was detected as occurring at the point for the transition from exhalation to inhalation. Importantly, these experiments performed with a subset of the mice showed that the application of light was on purpose performed randomly out of register with sniffing (see Fig. 2 of Li et al., 2014). 
A

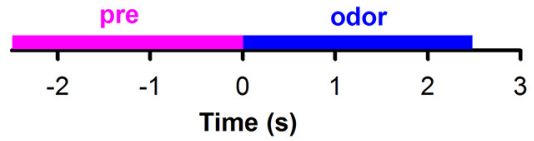

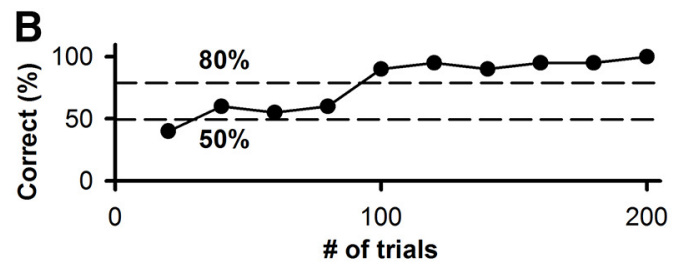

C

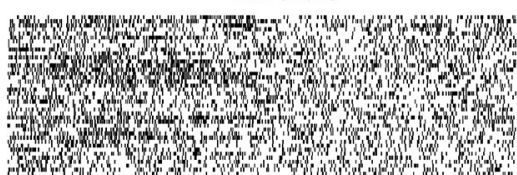

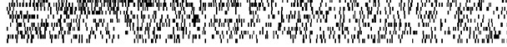

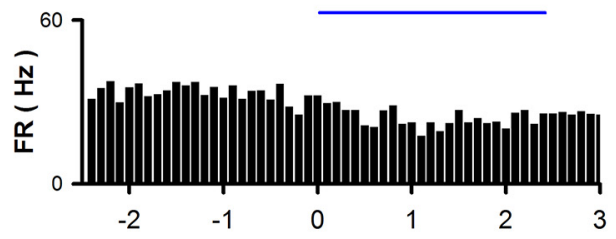

D-1

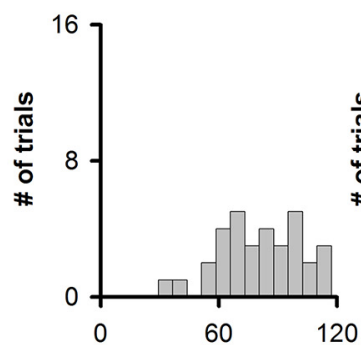

D-2

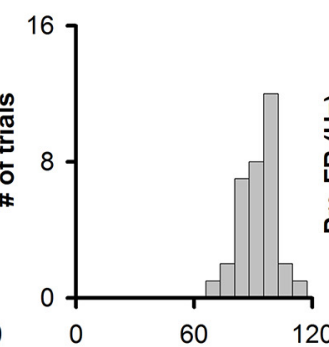

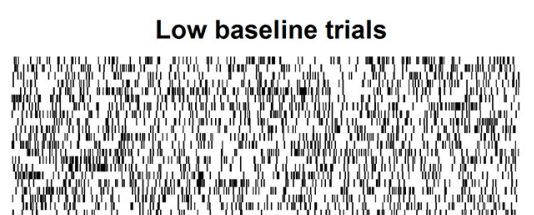

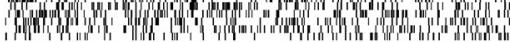

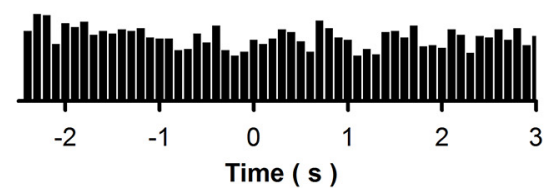

D-3

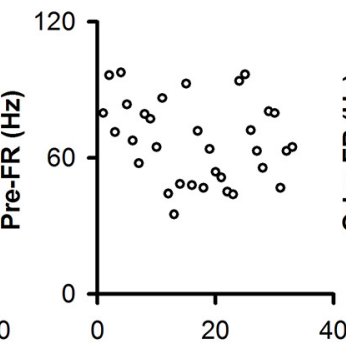

D-4

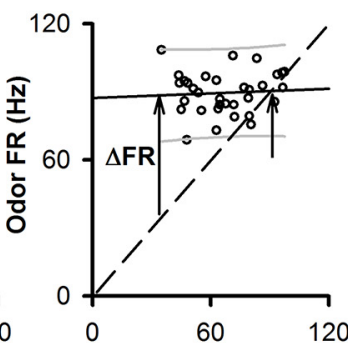

High baseline trials
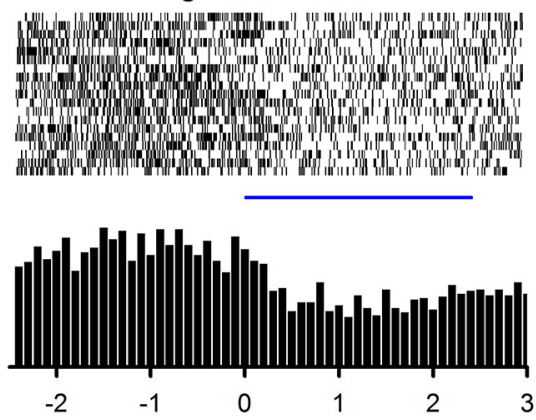

E-1

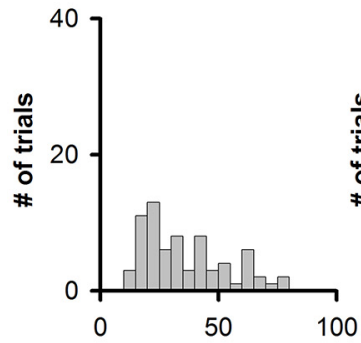

$\mathrm{E}-2$

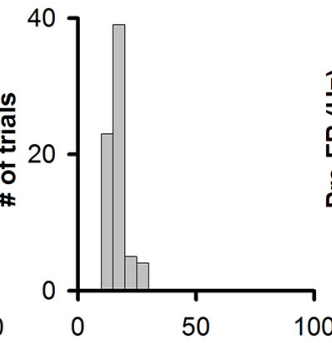

E-3

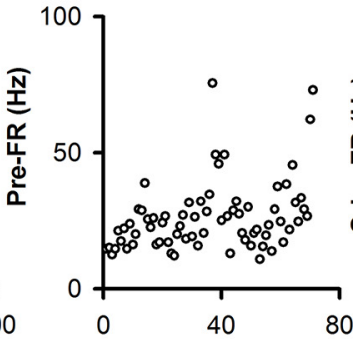

E-4

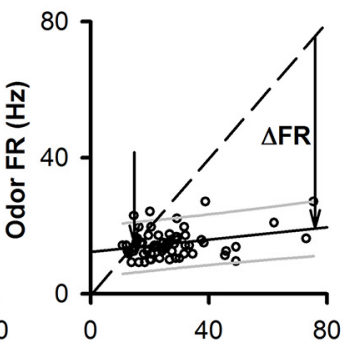

D-5

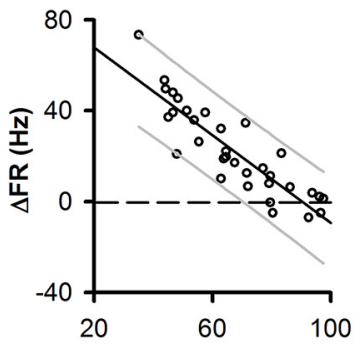

F-1

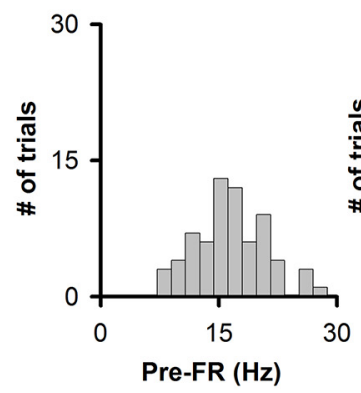

F-2

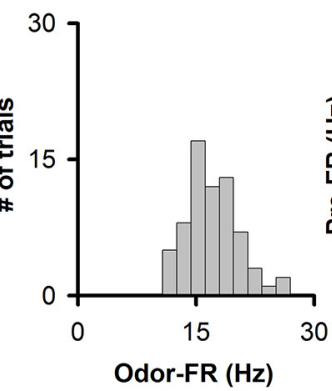

F-3

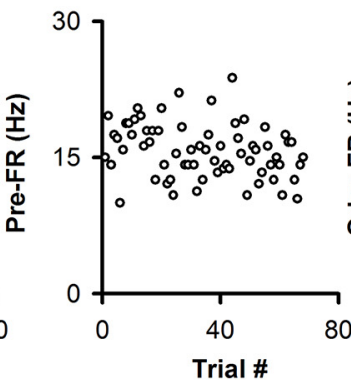

F-4

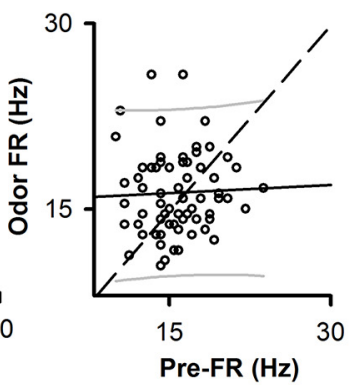

E-5

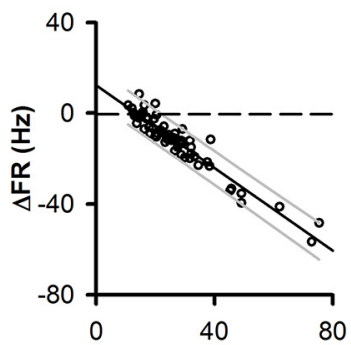

Figure 4. Examples in the go-no go task of the linear dependence of the trial-to-trial variability of the odorant-induced change in FR on the pre-FR in a subset of M/T cells. $A$, Time course for each trial. Pre-FR is defined as the FR for $2.5 \mathrm{~s}$ before addition of the odorant (pre), and odor-FR is the FR for $2.5 \mathrm{~s}$ during exposure to the odorant (odor). $\boldsymbol{B}$, Example of behavioral performance during a session of 200 trials in the go-no go task. Percent correct: percent of trials when the animal responded correctly in blocks of 10 rewarded/10 unrewarded odorant trials. $\boldsymbol{C}$, Example of odorant responses for a SU. Top, Raster plots (bottom to top: first to last trial). Bottom, PSTH. Left to right, All, low and high pre-FR trials (high pre-FR $\geq$ mean pre-FR). $D-F$, Examples of the dependence of odor FR on pre-FR. D1-F1, Histogram of mean per-trial pre-FRs for the entire go-no go session. D2-F2, Histogram of mean per-trial odor-FRs for the entire go-no go (Figure legend continues.) 
Offline spike clustering and classification a single units or multi units. Data were filtered digitally with a Butterworth filter from 1000 to $3000 \mathrm{~Hz}$ for spike detection using the MATLAB filtfilt function with zero phase shift. Custom software written in MATLAB was used to threshold spikes in each channel at 3 times the SD. Every thresholded spike ( 24 points at $24 \mathrm{kHz}$ ) was imported into a second program where we clustered the waveforms of similar shape by performing wavelet decomposition and superparamagnetic clustering using the method and MATLAB software used in previous studies (Doucette and Restrepo, 2008; Doucette et al., 2011; Li et al., 2014, 2015) (Fig. 1). A unit was classified as a single unit (SU) based on the criterion that fewer than $0.75 \%$ of the interspike intervals (ISIs) were $<1 \mathrm{~ms}$ (Jeanne et al., 2013; Stubblefield et al., 2015). This results in unimodal pre-FR distributions regardless whether recordings were with multielectrode arrays or tetrodes (Fig. 2).

For the go-go experiment, we recorded from 150 units in 5 mice. For the light-evoked stimulation experiment, we recorded from 83 units in 9 mice. Finally, for the go-no go experiment, we recorded with tetrodes from 595 units in 20 mice and from multielectrode arrays in 1163 units in 8 mice. With the exception of the pre-FR correlation analysis, where it was useful to determine whether the analysis was different for SUs and MUs, all analyses were performed with data for SUs.

Analysis of odor-elicited changes in FR and multiple comparison correction. Analysis of the difference in pre-FR between units recorded in different behavioral tasks and the dependence of changes in the FR upon stimulation with odorant were performed using custom-written MATLAB code. To test the difference between two datasets, we used Student's $t$ test when the null hypothesis of normal distribution was not rejected with the Anderson-Darling test, and the data values were expressed as mean \pm SEM. When the null hypothesis of normal distribution was rejected, the two datasets were compared using a Mann-Whitney $U$ test (MWT), and the data were displayed as a boxplot.

All statistical tests involving multiple comparisons were corrected using the false discovery rate (FDR) method (Curran-Everett, 2000). This included $p$ value correction for $t$ tests or MWT for difference between datasets (i.e., the difference in pre-FR between rewarded and unrewarded odorants for all SUs recorded in the go-no go task), tests for linearity or the difference between the measured slope and zero performed using best line fits for multiple SUs, tests of significance for the correlation coefficient for all unit pairs and multiple pairwise comparisons using the Kolmogorov-Smirnov or $\chi^{2}$ tests. Importantly, FDR with pairwise tests is suitable for testing significant differences in large datasets, can be used to correct $p$ values obtained with different tests (e.g., $t$ test and MWT), and does not require independent data (Curran-Everett, 2000; Benjamini and Yekutieli, 2001, 2005). This is necessary for analysis of neuronal recording with correlated firing between units whose pre-FR may or may not follow a normal distribution. The pFDR and the number of comparisons $(n)$ are reported for all tests with multiple comparison corrections, the test yields a significant difference when $p$ values $\leq$ pFDR.

Statistical test for a linear relationship between a significant odor-induced change in FR and pre-FR. We performed a simulation to gauge how

\section{$\leftarrow$}

(Figure legend continued.) session. D3-F3, Trial-per-trial variability for pre-FR through the session. D4-F4, Dependence of odor FR on pre-FR. Dashed line indicates odor FR $=$ pre-FR. The slope of the best-fit line was statistically different from 1 for $\mathbf{D} 4$ and $\boldsymbol{E 4}$, but not for $\boldsymbol{F}$. The difference of the slope from 1 was tested using significance for best linear fit corrected for multiple comparisons by calculating the critical significance level of the false discovery rate (pFDR) (Curran-Everett, 2000) as explained in Materials and Methods (D3,E3: $p \leq \mathrm{pFDR}=0.0055$, $n=1451$ SU-odor pairs). A statistical test of linearity corrected for multiple comparisons indicated that $\mathbf{D} \mathbf{4}$ and $\mathbf{E}$, but not $\mathbf{F}$, were linear (significant $p$ values $<\mathrm{pFDR}$, pFDR degrees $=$ $0.028, \mathrm{pFDR}_{-45 \text { degrees }}=0.055, n=1451 \mathrm{SU}$-odor pairs; for details on statistical test for linear dependence, see Materials and Methods and Fig. 3). D4, E4, Unlabeled arrow indicates the point where the best-fit line and odor $F R=$ pre-FR cross. $\triangle F R$ is the odor-induced change in $F R$, which is shown at a specific pre-FR in D4 and E4. Best-fit slope and intercept (Hz): D4, 0.034, $87.1 ; E 4,0.09,12.3 ; \boldsymbol{F} 4,0.043,15.6$. D5-F5, Dependence of $\Delta F R$ on pre-FR. Solid line indicates a best fit of the data. Dashed line indicates $\Delta F R=0$. Thin lines above and below the solid line are 5th and 95th percentile estimates. Best-fit slope and intercept (Hz): D5, $-0.97,87.1 ; \mathbf{E 5}$, $-0.91,12.3 ; F 5,-0.96,15.6$. suitable different approaches were to perform a statistical test of whether there is a linear dependence between a statistically significant odorinduced change in FR and pre-FR. Figure $3 A-D$ shows examples of different dependences between odor FR and pre-FR for simulated data where we resampled the pre-FR distribution for the SU-odor pair shown in Figure 4F1. The cumulative probability for the pre-FR was resampled by generating random numbers in the $0-1$ interval that were used to choose different FR values corresponding to that particular probability.

We first asked the question whether it was suitable to study directly the relationship between the change in FR elicited by the odor $(\Delta \mathrm{FR})$ and pre-FR. Figure $3 A 2$ shows an example of this relationship for a simulation where there is not an odorant response: both odor FR and pre-FR were drawn by random sampling of the pre-FR distribution measured for the $\mathrm{SU}$-odorant pair in Figure $4 F 1$ and $\Delta \mathrm{FR}$ was calculated $(\Delta \mathrm{FR}=$ odor FR - pre-FR). Analyzing data generated by this simulation made it clear that this is a problematic approach because in this case there is a linear relationship between $\Delta \mathrm{FR}$ and pre-FR, yet there is no odorant response (Fig. 3A2). This linear relationship is generated because of a simple reason: when a pre-FR below the mean FR is drawn from the distribution, the odor FR randomly drawn from the same distribution is more likely to be above the pre-FR (and vice versa). Thus, if we had chosen to perform the analysis of the $\Delta \mathrm{FR}$ versus pre-FR relationship, we would have to limit the analysis to the $\mathrm{SU}$-odorant pairs whose FR is stable within-trials to avoid classifying an odor-induced change in FR as linearly dependent on pre-FR when in reality there is no response to the odorant. We did perform that analysis, and we obtained results similar to those in this publication (data not shown). But that approach is not as robust as the analysis described below because a statistical test for within-trial stability of FR yields a false rejection for a substantial number of SU-odorant pairs due to the combination of the short time interval $(2.5 \mathrm{~s})$ and the large inherent variance of pre-FR.

We then turned our attention to an analysis of the relationship of odor FR versus pre-FR to develop a valid statistical test for a linear dependence of an odor-induced change in FR on pre-FR. Data were considered linearly dependent if the relationship was fit by a line, regardless whether the slope is equal to 0 . Furthermore, the odorant-induced change in FR was considered significant if the dependence of odor FR on pre-FR was linear with a slope different from 1 . It is important to state that finding that there is an odorant response where odor FR depends linearly on pre-FR with a slope different from 1 necessarily implies that there is also a linear dependence of the odorant-induced change in FR $(\Delta \mathrm{FR})$ on pre-FR because $\Delta \mathrm{FR}=$ odor FR - pre-FR (compare Fig. $3 C 1, D 1$ with Fig. $3 C 2, D 2)$. In contrast, when odor FR = pre-FR, the linear relationship has a slope of 1 . In this case, there is not an odorant-induced change in FR $(\Delta \mathrm{FR}=0$; compare Fig. $3 B 1$ with Fig. $3 B 2)$

To test for significance of linearity of the odor FR versus pre-FR relationship, we tested for significance of the F-statistic of the linear regression model versus a constant model using MATLAB fitlm (linear regression fit). We proceeded to evaluate the result of this fitlm test of linearity for relationships where odor FR is generated with a linear relationship in the presence/absence of an odorant-induced response. When there is no odorant response, but there is a linear relationship odor FR = pre-FR (with added random normal noise of a magnitude of one-fourth of the $\mathrm{SD}$ of the pre-FR distribution, $\Delta \mathrm{FR}=0$ ) (Fig. $3 B, F$, gray bar $\mathrm{B}$ ), the test of the $F$ statistic of the linear regression was significant as expected for this linear dependence. However, the $F$ test for linear best fit of odor FR versus pre-FR does not yield a significant $p$ value for a linear dependence where odor FR values fall on a line parallel to the horizontal pre-FR axis, where there is a clear odorant-induced $\Delta \mathrm{FR}$ (Fig. $3 C, D, F$, gray bars $\mathrm{C}$, $D)$. This happens because this $F$ statistic test determines whether the linear regression coefficients are 0 with the exception of the coefficient for the intercept.

Because of this, a single fitlm test of linearity was not sufficient to classify correctly all odor FR versus pre-FR datasets as linearly dependent. However, we found that, if we performed this fitlm test after a 45 degree clockwise rotation of the odor FR versus pre-FR axis, $p$ values for the $F$ test were significant for horizontal odor FR versus pre-FR data (Fig. $3 C, D, F$, slanted line bars $C$ and $\mathrm{D}$ ). Thus, to establish whether the data for odor FR versus pre-FR followed a linear dependence, regardless of 

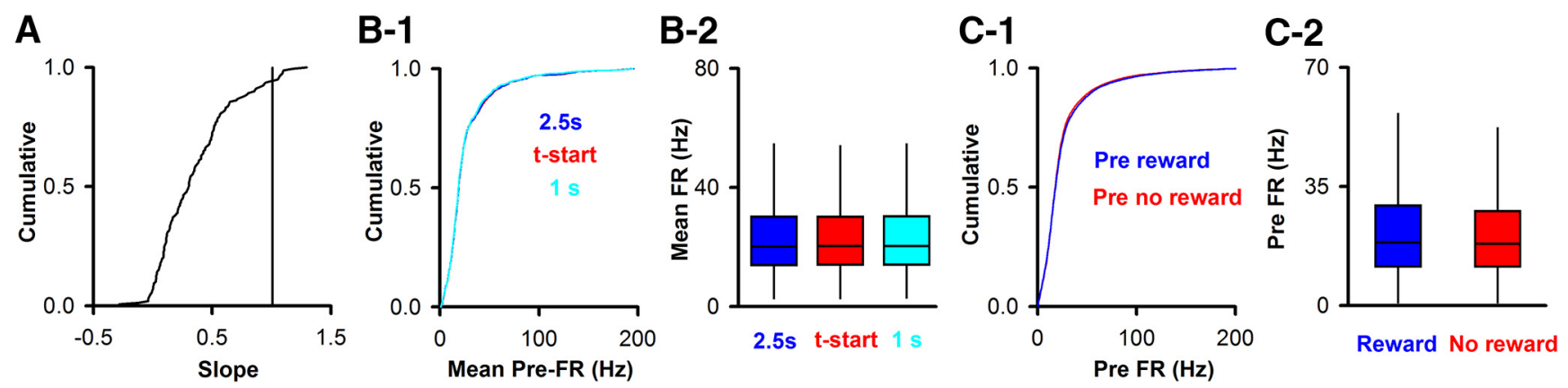

Figure 5. Slope and pre-FR for the dependence of the FR in the presence of the odorant on the pre-FR for the go-no go sessions. $\boldsymbol{A}$, Cumulative histogram for the slope of odor-FR versus pre-FR for SU-odor pairs that displayed a linear relationship with a slope significantly different from 1 ( $n=159)$. $\boldsymbol{B}$, The pre-FR did not differ when it was calculated for different intervals before the addition of the odorant. The pre-FR is shown calculated for intervals of 2.5 or $1 \mathrm{~s}$ before odor addition, or for calculation of pre-FR during the time from odor port entry to odor addition that varies randomly from 1 to 1.5 s in each trial ( $t$-start). B1, Cumulative histogram. Pairwise threefold comparison with Kolmogorov-Smirnov, $p>0.99, \mathrm{pFDR}=0.05, n=735$ SUs. B2, Box-and-whisker plot with MWT, $p>0.7, p F D R=0.05, n=735$. C, Dependence of pre-FR on whether the animal was or was not rewarded in the previous trial. C1, Cumulative histogram. Kolmogorov-Smirnov, $p<0.0001$, $n=735$ SUs. C2, Box-and-whisker plot. MWT, $p=0.002, n=735$.

whether odor FR points fall on a horizontal line, we performed the test of linear best fit with both the original odor FR versus pre-FR orientation and after a 45 degree clockwise rotation of the axes (dual-orientation linearity test). Figure $3 F$ (black bars B-D) shows that this test classifies the dependencies shown in Figures $3 B-D$ as linear.

In contrast, this dual-orientation linearity test did not classify as linear the odor FR versus pre-FR relationship when both odor FR and preFR were drawn randomly from the pre-FR distribution (no odorantinduced change in FR). The example in Figure $3 A 1$ shows the odor FR versus pre-FR relationship corresponding to the $\Delta$ FR versus pre-FR shown in Figure $3 A 2$ where odor FR was generated by random resampling of pre-FR in the absence of an odor response. A dual-orientation test for linearity was not significant for the example in Figure $3 A 1$ and for the large majority of the $1451 \mathrm{SU}$-odorant pairs when both odor FR and pre-FR were randomly drawn (Fig. $3 F$, black A bar).

Therefore, to test whether there is a linear dependence of an odorinduced change in FR on pre-FR, the linear relationship of odor FR on pre-FR was tested with the dual-orientation linearity test, and we tested whether the slope of this dependence was different from 1 using MATLAB fitlm. These tests were performed for all SU-odor pairs (1451 comparisons for go-no go) for the different odor FR versus pre-FR simulations with examples shown in Figure $3 A-D$. The percent of slopes different from 1 and the percent of linear odorant FR versus pre-FR relationships are shown for the different simulations in Figure $3 E$ and Figure $3 F$, respectively. The $p$ values were corrected for multiple comparisons using FDR (Curran-Everett, 2000) that yields a critical significance level ( $\mathrm{pFDR}$ ). For the linearity test we provide the pFDR for the original axis orientation for odor FR versus pre-FR $\left(\mathrm{pFDR}_{0 \text { degrees }}\right)$ as well as for the calculation after a -45 degree rotation $\left(\mathrm{pFDR}_{-45 \text { degrees }}\right.$ ). This analysis shows that this statistical test of linearity in two different orientations performs a robust test of whether an SU-odorant pair responds to an odorant and shows a linear dependence of the odor FR versus pre-FR dependence (and by default of the $\Delta \mathrm{FR}$ vs pre-FR dependence).

\section{Results}

To test the hypothesis that the odorant-induced change in FR of $\mathrm{M} / \mathrm{T}$ cells changes as a function of pre-FR, we recorded from cells in the mitral cell layer in awake behaving mice. To determine whether this dependence on pre-FR differs based on behavioral status, we trained mice to lick to obtain a water reward regardless of the odorant presented (passive, go-go task) or when only one of two odorants was rewarded (active, go-no go task). In addition, we separately examined trials where the animals made mistakes in the active odorant discrimination task.

\section{Are odorant FR responses dependent on the pre-FR in the active go-no go odor discrimination task?}

We recorded extracellular voltage changes generated by action potentials in the mitral cell layer of the $\mathrm{OB}$ in the go-no go odor discrimination task (Doucette et al., 2011; Li et al., 2015). In each behavioral training session, the animal was exposed to one of two odorants in 50-200 trials (time course per trial shown in Fig. 4A). In the active go-no go session, the animal learned to respond to the rewarded odorant, but not to the unrewarded stimulus (Fig. $4 B)$. Figure $4 C$ shows an example of the response of an SU to the rewarded odorant ( $1 \%$ phenyl acetate). When all the trials are shown, a change in the FR elicited by the odor is not apparent (Fig. 4C, left). However, when the trials were sorted into low/high pre-FR (low pre-FR $\leq$ mean pre-FR), there appears to be a decrease in FR elicited by the odorant for the high pre-FR trials (Fig. $4 C$, right), but not for the low pre-FR trials (Fig. $4 C$, left). This suggests that the change in FR elicited by the odorant depends on the pre-FR.

To perform a valid analysis of the potential dependence of the response to the odorant on pre-FR, we performed statistical tests to determine whether the dependence of FR in the presence of the odorant on pre-FR was linear with a slope different from 1. As explained in the Materials and Methods, this results in a statistically reliable determination whether there is a linear dependence of the change in FR elicited by the odorant as a function of pre-FR (Fig. 3). The FR in the presence of the odorant (odor-FR) and pre-FR were calculated for each trial as the FR for $2.5 \mathrm{~s}$ during (odor FR) or before (pre-FR) odor addition (Fig. 4A). A linear dependence for odor-FR versus pre-FR with a slope equal to 1 (odor-FR = pre-FR) does show that odor FR depends linearly on pre-FR, but indicates that the SU did not respond to the odorant $(\Delta \mathrm{FR}=0$, where $\Delta \mathrm{FR}=$ odor-FR pre-FR). A slope significantly different from 1 for this linear dependence is evidence of dependence of the odorant-induced change in FR $(\Delta$ FR) on pre-FR (Fig. 3). The statistical significance of the linear dependence was assessed by tests of the significance of a linear bestfit determined under different orientations of the odor FR versus pre-FR axes ( 0 degree rotation and -45 degree rotation). The use of the two tests ensures that linear dependence is tested correctly regardless of the value of the slope of odor-FR versus pre-FR, as shown for simulated data in Figure 3 and explained in Materials and Methods. $p$ values were corrected for multiple comparisons by calculating the critical significance level of the false discovery rate (pFDR) (Curran-Everett, 2000) (significant $p$ values $\leq$ pFDR). 
A

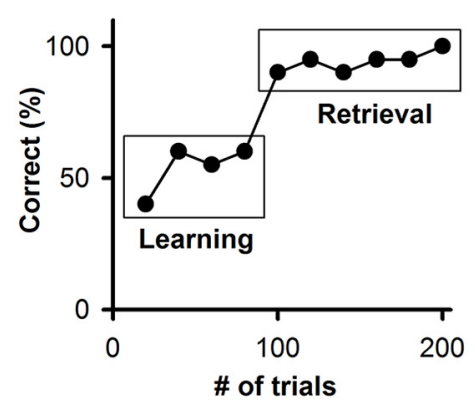

C

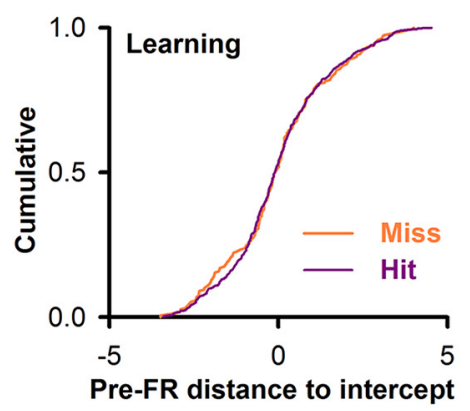

E

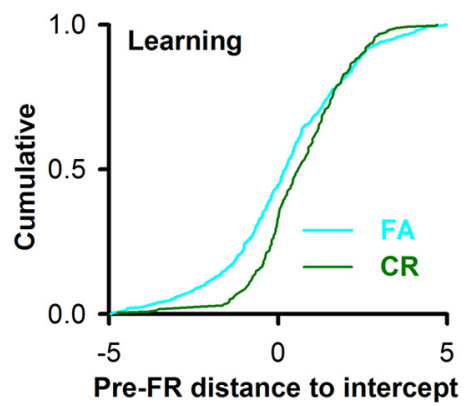

B

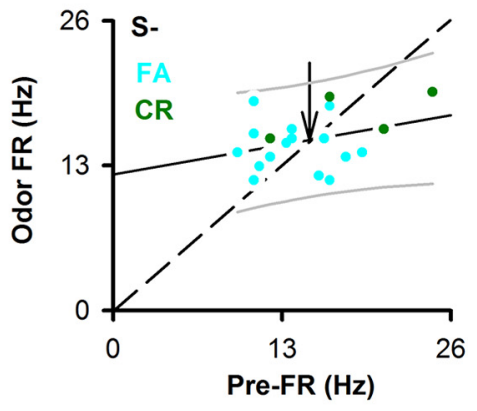

D

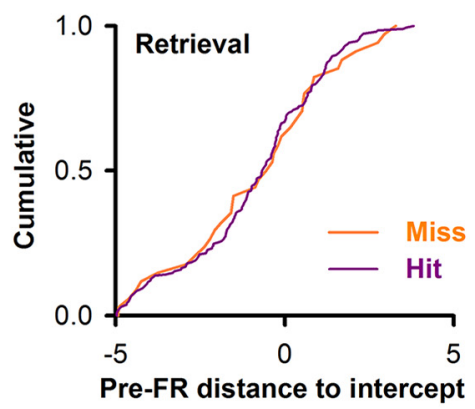

$\mathbf{F}$

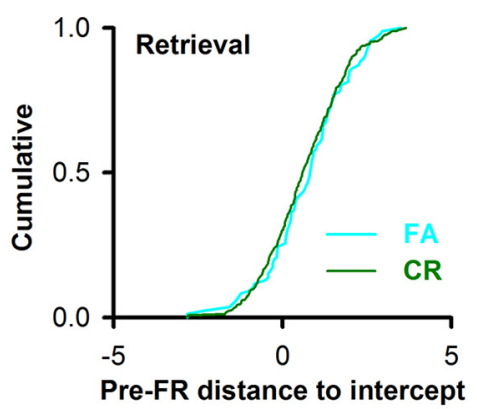

Figure 6. The pre-FR differs between correct rejections and FAs when the animal is learning to differentiate between the two odorants. $\boldsymbol{A}$, Example of behavioral performance during a session of 200 trials in the go-no go task. Percent correct: percent of trials when the animal responded correctly in blocks of 10 rewarded/10 unrewarded odorant trials. Learning is the segment for percent correct $\geq 45 \%$ and $\leq 65 \%$. Retrieval is the segment for percent correct $\geq 80 \%$. B, Example for an SU-odor pair of the dependence of odor FR on pre-FR for the unrewarded odorant during the learning segment. Green represents CR. Light blue represents FA. This dependence tested significant for linearity and with a slope different from 1. Solid line indicates the best fit. Dashed line indicates odor FR = pre-FR. Thin solid lines above and below the best-fit line are 5th and 95th percentiles. Unlabeled arrow indicates the point where the best-fit line and odor $F R=$ pre-FR cross. Best-fit line slope and intercept: $0.2,12.3 \mathrm{~Hz}$. $\boldsymbol{C}-\boldsymbol{F}$, Cumulative probability for the distance along the pre-FR axis between a point and the intercept between the best-fit line and odor FR $=$ pre-FR. Dividing by the SD of pre-FR normalized this distance. C, Hits versus Miss for the learning segment (Kolmogorov-Smirnov, $p=0.82$, number of trials: 577 hits, 186 misses, pFDR $=0.04,28$ pairwise comparisons). $\boldsymbol{D}$, Hits versus Miss for the retrieval segment (Kolmogorov-Smirnov, $p=0.99$, number of trials: 190 hits, 34 misses, $\mathrm{pFDR}=0.04$ ). $\boldsymbol{E}$, (R versus FA for the learning segment (Kolmogorov-Smirnov, $p<0.0001$, number of trials: 233 CRs, $667 \mathrm{FAs}, \mathrm{pFDR}=0.04$ ). $\boldsymbol{F}$, CR versus FA for the retrieval segment (Kolmogorov-Smirnov, $p=0.63$, number of trials: 398 CRs, 86 FAs, pFDR $=0.04$ ).

This analysis was performed for $1451 \mathrm{SU}$-odor pairs recorded in the active go-no go task in two previous studies (Doucette et al., 2011; Li et al., 2015). Examples of the analysis of the dependence of Odor FR on pre-FR are shown for three SU-odor pairs in Figure $4 D-F$. For $61 \%$ of the SU-odor pairs, there was a linear dependence of odor FR on pre-FR (significant $p$ values $<$ pFDR, $\mathrm{pFDR}_{0 \text { degrees }}=0.028, \mathrm{pFDR}_{-45 \text { degrees }}=0.055, n=1451 \mathrm{SU}$ odor pairs). In addition, for a subset of the SU-odor pairs (11\%, $n=159$ of 1451 ), there was a dependence of the $\Delta \mathrm{FR}$ on pre-FR as evidenced by a slope of the best-fit line for odor FR versus
pre-FR statistically different from 1 ( $p \leq$ $\mathrm{pFDR}=0.0055, n=1451$ SU-odor pairs). Examples for this linear dependence with a slope different from 1 for odor FR versus pre-FR are shown in Figure 4D, E. For the SU-odor pair in Figure 4D4, the odorant elicited a 2.5-fold increase in FR at a low pre-FR (Fig. $4 D 4$, arrow labeled “ $\Delta$ FR"), but there was no change in FR at high pre-FR where the best-fit line intercepts with the odor FR = pre-FR line (Fig. 4D4, unlabeled arrow). On the other hand, for the SU-odor pair in Figure 4E, the odorant elicited a fourfold decrease in FR at high pre-FR, but not at low pre-FR (Fig. $4 E 4$, arrows labeled " $\Delta \mathrm{FR}$ " and unlabeled arrow; the raw data are shown in Fig. $4 C$ ). In contrast, for $39 \%$ of the SU-odor pairs the dependence of odor FR on pre-FR was not linear as expected for random distribution of odor FR and pre-FR (an example is shown in Fig. $4 F$ and data generated by random resampling of pre-FR are shown in Fig. 3A).

Thus, in $11 \%$ (159) of the 1451 SUodor pairs, there is significant trial-to-trial variability in pre-FR and there is a significant change in FR elicited by the odorant whose magnitude is linearly dependent on pre-FR. For the majority of these pre-FRlinear odor-responsive SU-odor pairs the odor-elicited change in FR decreased as a function of pre-FR, and the slope of odor FR versus pre-FR was $<1$ (Fig. 5A). The value of pre-FR did not change when FR was determined by calculating FR using time intervals ranging between 1 and $2.5 \mathrm{~s}$ before the time when the odorant was presented (Fig. 5B1, pairwise threefold comparison with Kolmogorov-Smirnov, $p$ value $>0.99, \mathrm{pFDR}=0.05, n=735 \mathrm{SUs}$, Fig. 5B2, MWT, $p$ value $>0.7$, pFDR $=$ $0.05, n=735)$. For the rest of the study, pre-FR was calculated for $2.5 \mathrm{~s}$ before odor addition. Finally, we asked whether the pre-FR was different depending on whether the animal was or was not rewarded in the previous trial. There was a very small, but statistically significant, difference between pre-FR depending on the outcome of the previous trial (Fig. 5C1, Kolmogorov-Smirnov, $p<0.0001, n=$ 735 SUs; Fig. 5C2, MWT, $p=0.002, n=$ 735). The magnitude of this change is small; therefore, we do not consider this to be a relevant finding.

The pre-FR differs between correct rejections and false alarms (FAs) when the animal is learning to differentiate between odorants in the active go-no go task

We surveyed the difference in pre-FR when animals made mistakes in a subset of trials in the go-no go task using the data in experiments published previously (Doucette et al., 2011; Li et al., 2015). Figure 6A shows an example of the performance of a mouse learning to differ- 
A

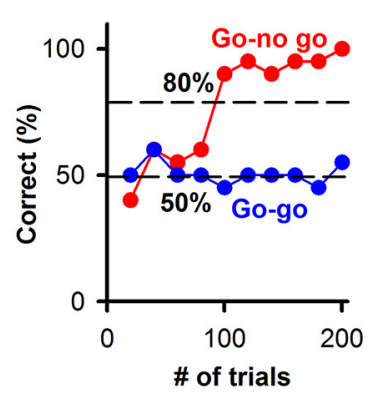

C-1

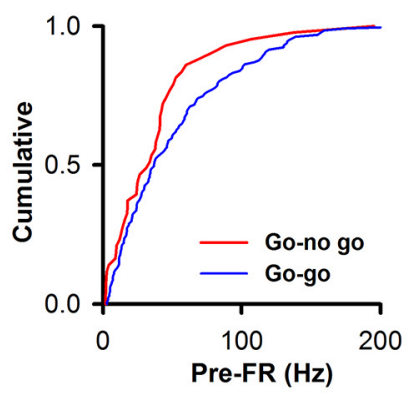

F-1

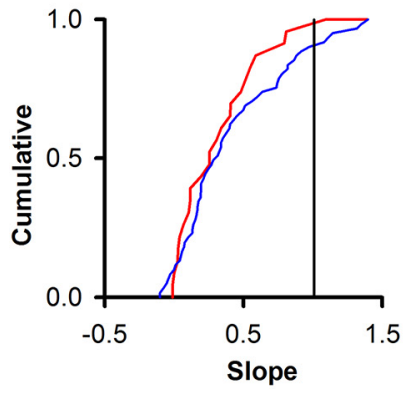

B-1

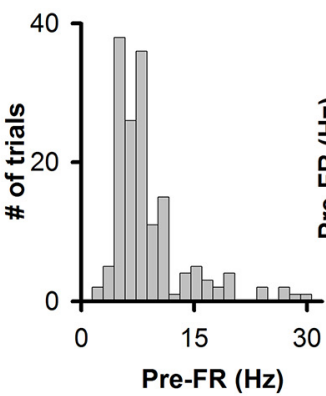

C-2

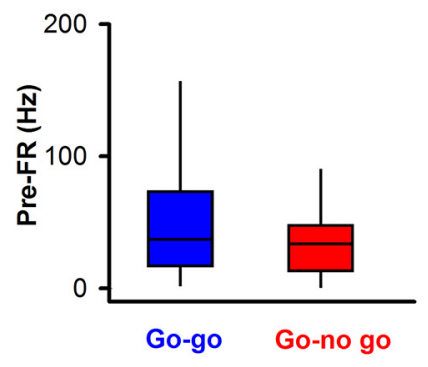

F-2

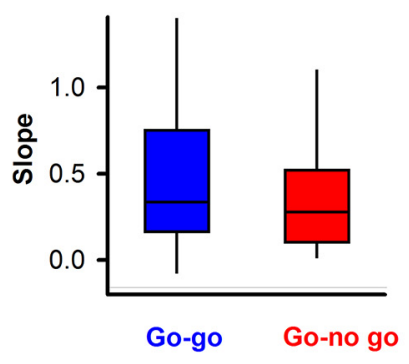

B-2

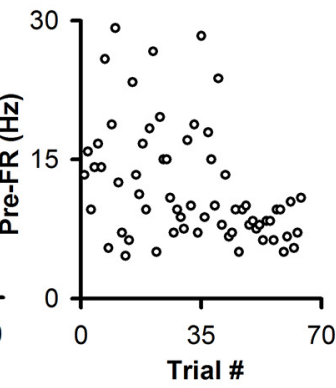

D

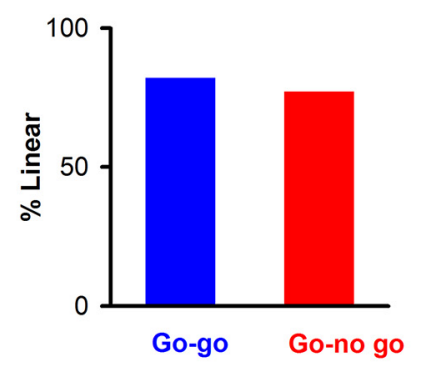

G-1

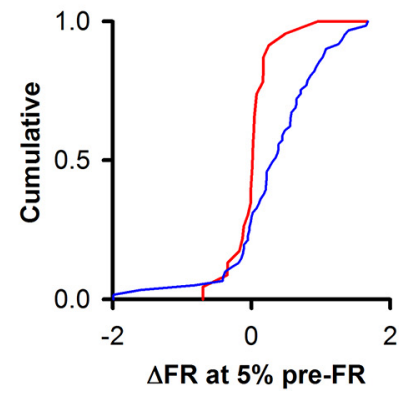

B-4

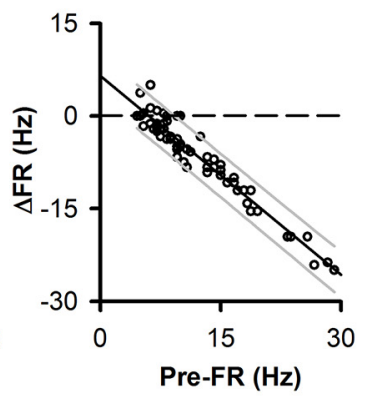

E

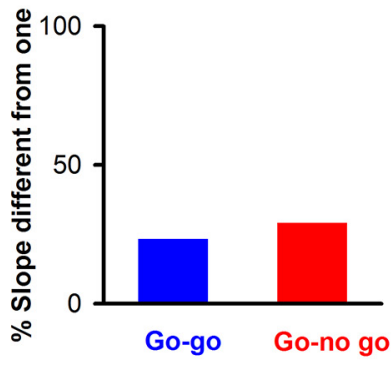

Figure 7. Differences in the dependence of odor FR on pre-FR between the go-no go and go-go tasks. $A$, Examples of behavioral performance during the go-no go and go-go tasks. Percent correct: percent of trials when the animal responded correctly in blocks of 10 rewarded/10 unrewarded odorant trials. $\boldsymbol{B}$, Example of the dependence of odor FR on pre-FR for an odor-SU pair recorded during a go-go task where both odorants were rewarded. B1, Histogram of per-trial pre-FRs for the entire go-go session. B2, Trial-per-trial variability for pre-FR through the session. B3, Dependence of odor FR on pre-FR. Dashed line indicates odor $F R=$ pre-FR. The slope of the best-fit line was statistically different from 1 ( $p$ value $\leq \mathrm{pFDR}=0.01, n=300$ SU-odor pairs), and a statistical test corrected for multiple comparisons indicated that the relationship was linear (significant $p$ values $<\mathrm{pFDR}, \mathrm{pFDR}_{0 \text { degrees }}=0.035, \mathrm{pFDR}-45$ degrees $=0.01, n=300$ SU-odor pairs; for details on statistical test for linear dependence, see Materials and Methods and Fig. 3). Best-fit slope and intercept: $-0.074,6.47 \mathrm{~Hz}$. B4, Dependence of $\Delta$ FR on pre-FR. Solid line indicates a best fit of the data. Dashed line indicates $\Delta$ FR $=0$. Thin lines above and below the solid line are 5th and 95th percentile estimates. Best-fit slope and intercept (Hz): -1.07, 6.47. C-G, Comparison between go-go and go-no go in SU data recorded from 4 mice that underwent both go-go and go-no go tasks. C, The mean pre-FRper session does not differ between the go-no go and the go-go tasks ( $n=43$ SUs in the go-no go task and 131SUs in the go-go task). C1, Cumulative probability (Kolmogorov-Smirnov, $p=0.12) .(2$, Box-and-whisker plot for pre-FR (MWT, $p=0.1)$. D, The percent of SU-odor pairs displaying a linear dependence of odor FR versus pre-FR does not differ between go-no go and go-go tasks $\left(\chi^{2}\right.$, $p>0.05)$. $\boldsymbol{E}$, The percent of SU-odor pairs displaying a linear dependence with a slope different from 1 does not differ between go-no go and go-go tasks $\left(\chi^{2}, p>0.05\right)$. $\boldsymbol{F}$, The distribution of the slopes of the odor FR versus pre-FR dependence does not differ between go-no go and go-go tasks. F1, Cumulative histogram (Kolmogorov-Smirnov, $p=0.62$ ). F2, Box-and-whisker plot (MWT, $p=0.44$ ). G, The change in FR elicited by the odorant at five percentile pre-FR (normalized by dividing by the SD of pre-FR) differs between go-no go and go-go. G1, Cumulative histogram (Kolmogorov-Smirnov, $p=0.0002$ ). G2, Box-and-whisker plot (MWT, $p=0.005)$. *Statistically significant.

entiate between two odorants in this active go-no go task. The pre-FR was compared between the period when the mouse is learning to differentiate the odorants (learning segment, percent correct $<65 \%$; Fig. $6 A$ ) and the period when the mouse has learned to differentiate between odorants and is retrieving this information to engage in the correct sustained licking response (retrieval segment, percent correct $>80 \%$, Fig. $6 A$ ). Pre-FR was computed for trials when the animal responded correctly by licking to the rewarded odorant (Hits) or refrained from licking for the unrewarded odorant (correct rejections [CRs]), or when the animal was responding incorrectly by licking for the unrewarded odorant (FA) or not licking for the rewarded odorant (Miss). Figure $6 B$ shows an example, for a single M/T SU, of the relationship between odor FR and the pre-FR for the unrewarded odorant during the learning segment of the behavioral session. Most of the CR points display pre-FR larger than the intercept of the best-fit line with the odor FR = pre-FR line. Interestingly, this trend for pre-FR in CR trials holds when we surveyed the data for all $\mathrm{M} / \mathrm{T}$ units. The distance along the pre-FR axis from the pre-FR for each data point to the intercept of the best-fit line with the odor FR = pre-FR line (pre-FR distance to intercept) for FAs tended to fall below the pre-FR distance to intercept for the CRs during the learning segment (Fig. 6E, Kolmogorov-Smirnov, $p<0.0001$, number of trials: 233 CRs, 667 FAs, pFDR $=0.04)$. In contrast, the cumulative probability for the pre-FR distance to inter- 
A
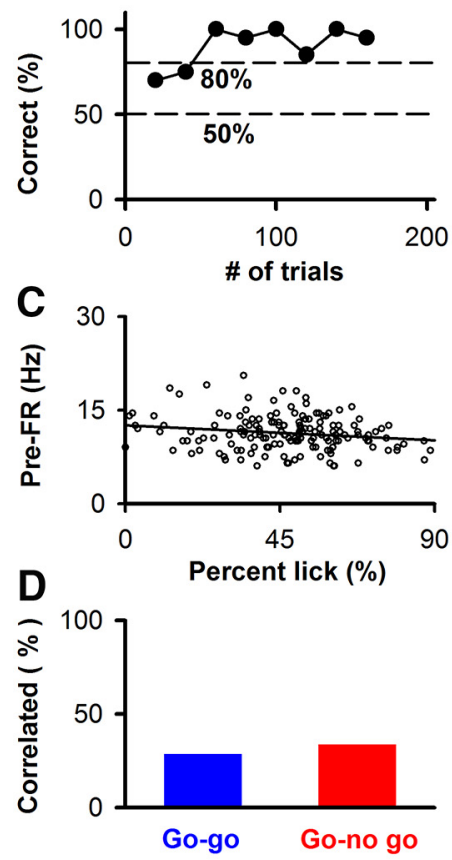

B-1

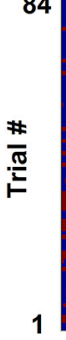

$\frac{\#}{*}$

pre-odor $(2.5 \mathrm{~s}) \quad$ odor $(2.5 \mathrm{~s})$

\section{B-2}

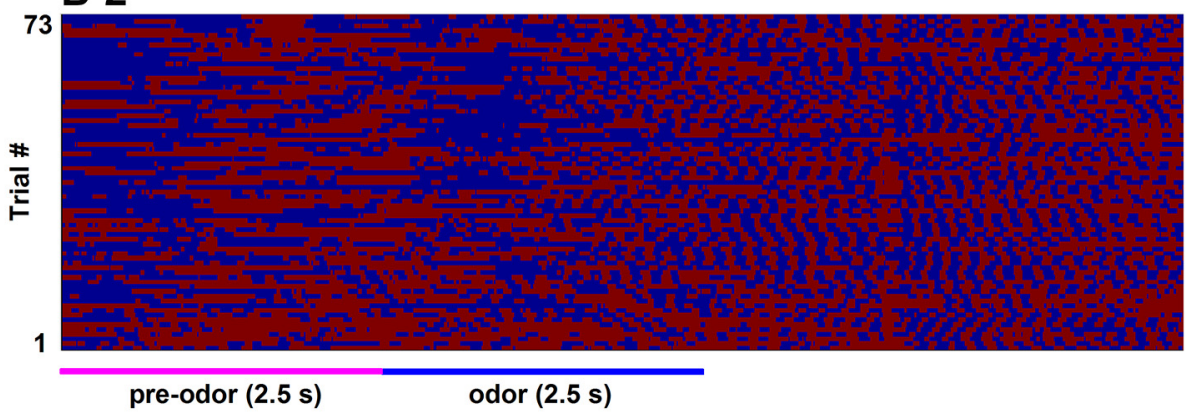

E

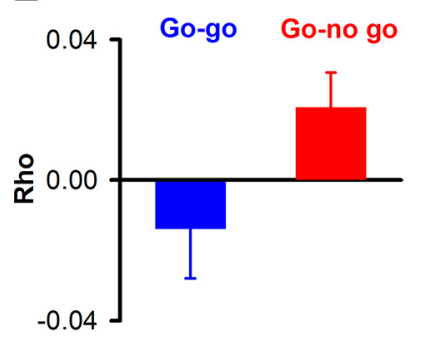

F

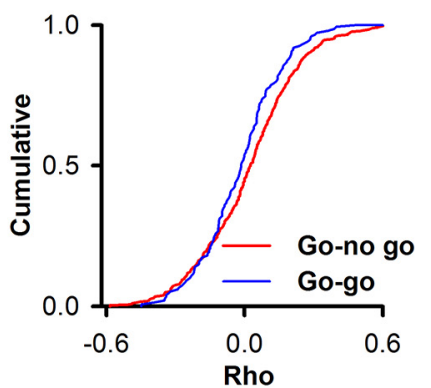

G

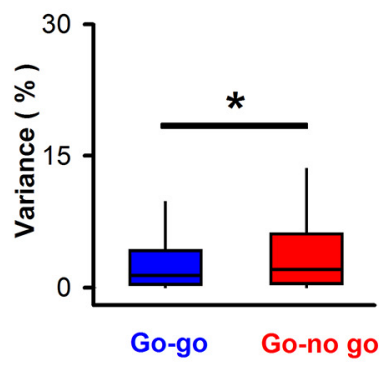

H

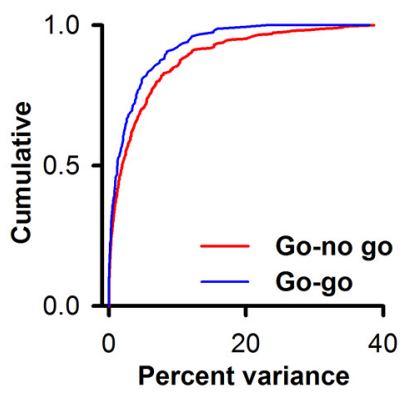

Figure 8. Dependence of prestimulus FR on licking. $A$, Performance of a mouse in a go-no go experiment. $\boldsymbol{B}$, Trial per trial time course for licks for the experiment whose behavioral performance is shown in $A$. Red represents tongue touches the lick tube. $C$, Example of the relationship between pre-FR and the percent of the time that the mouse licks in the preodor period (percent lick) for an SU recorded from in this session. Line indicates the best fit (slope $=-0.027$, intercept $=12.6)$. The correlation coefficient $(-0.18)$ was not significant $(p=0.024>\mathrm{pFDR}=0.017, n=383 \mathrm{SU}$ s for go-no go). Best-fit slope $-0.02 \mathrm{~Hz} / \%$, intercept $12.7 \mathrm{~Hz}$. D, Percent of SUs that display a statistically significant correlation between pre-FR and percent lick (pFDR is 0.014 for 150 SUs in the go-go sessions, $\chi^{2}, p$ value for difference in percent correlation $>0.05$ ). E, Mean and SEM for the correlation coefficients for all SUs recorded from in the go-go and go-no go sessions (unpaired $t$ test, $p=$ 0.08). $\boldsymbol{F}$, Cumulative distribution of correlation coefficients (Kolmogorov-Smirnov, $p=0.04$ ). $\boldsymbol{G}, \boldsymbol{H}$, Percent of the variance of pre-FR that is accounted for by the dependence between pre-FR and percent lick (percent variance) determined for SUs that displayed significant correlation (129 SUs for go-no go and 43 SUs for go-go). G, Boxplot for percent variance (MWT, $p=0.04$ ). $\boldsymbol{H}$, Cumulative distributions for the percent variance (Kolmogorov-Smirnov, $p=0.15)$. *Statistically significant.

cept did not differ between CRs and FAs for the retrieval segment (Fig. $6 F$ ), or for Hits and Misses (Fig. 6C,D). Because of the linear relationship of odor FR on pre-FR, the fact that for the learning segment the pre-FRs for FA tend to fall below the intercept compared with pre-FR for CR indicates that the odorant-induced change in FR is larger for the FA trials in this segment. This difference between $\mathrm{CR}$ and FA suggests that differences in pre-FR convey information relevant to the behavioral response to the stimulus in the learning segment indicating that pre-FR may reflect an anticipatory cue.

\section{Differences between the active and passive behavioral tasks} for the dependence of the FR in the presence of the odorant on the pre-FR

In the active go-no go task, the thirsty mice learn to discriminate between an odorant that is rewarded with water from the unrewarded odorant (Doucette et al., 2011; Li et al., 2015). In contrast, in the go-go task, the animals are rewarded with water for licking regardless of which of the two odorants is presented (Li et al., 2015). This is a passive odor exposure task because the animals are not required to intentionally sample the odorants to obtain a reward. As a result, the animals learn to respond differentially to the odorants in the active task (Fig. $7 \mathrm{~A}$, red), but they respond equally to the two odorants in the passive task (Fig. 7A, blue). We analyzed data generated in our previous publication ( $\mathrm{Li}$ et al., 2015) for 4 mice that were tested in both the go-no go and go-go tasks. Figure $7 B$ shows an example of the linear dependence of odor FR on pre-FR for a SU-odor pair recorded in the go-go task. This relationship was linear and the slope differed from 1. Interestingly, a perusal of the experimental data revealed an apparent difference in the dependence of odor FR on pre-FR between the passive and active tasks. The mean pre-FR did not differ between the active and passive odorant tasks (Fig. $7 C$, Kolmogorov-Smirnov, $p=0.12$, MWT, $p=0.1, n=43$ SU-odor pairs for go-no go and 131 for go-go). In addition, the percent of SU-odor pairs that 
A

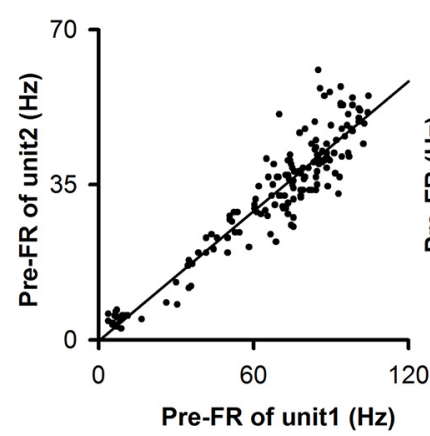

E

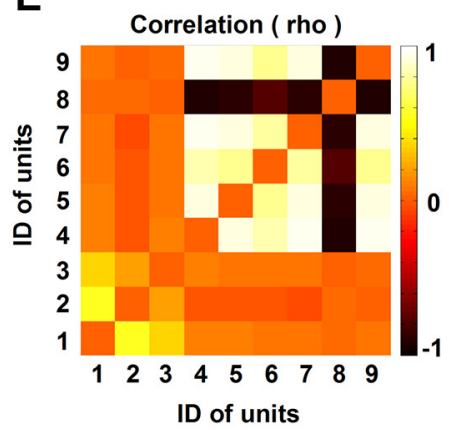

B
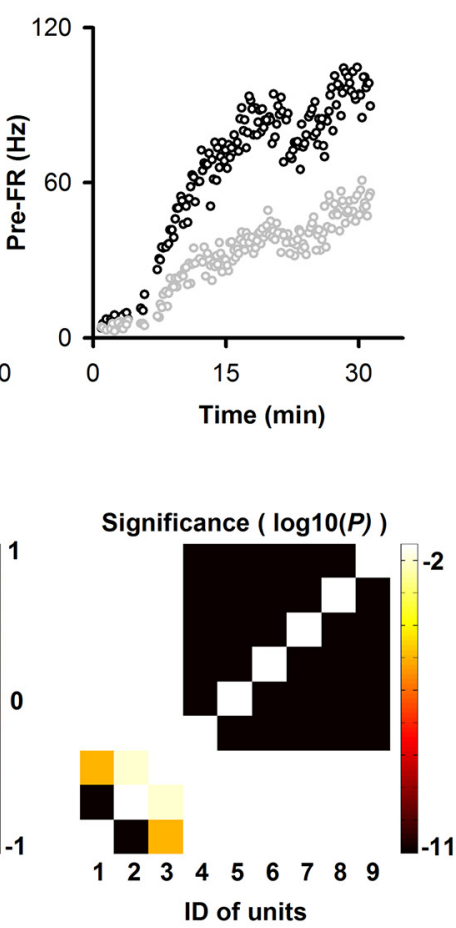

C

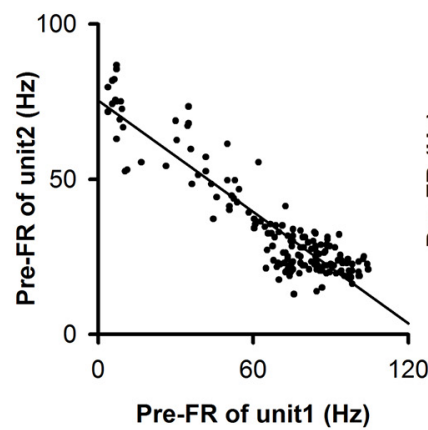

F

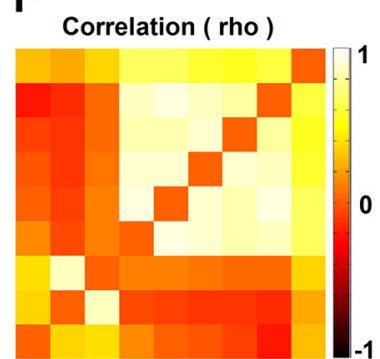

$\begin{array}{lllllllll}1 & 2 & 3 & 4 & 5 & 6 & 7 & 8 & 9\end{array}$ ID of units

H

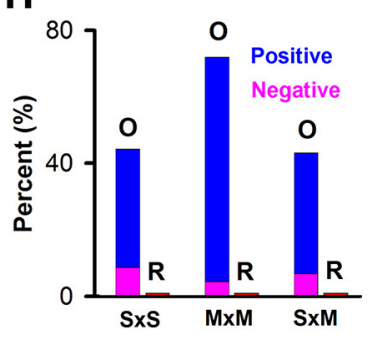

D

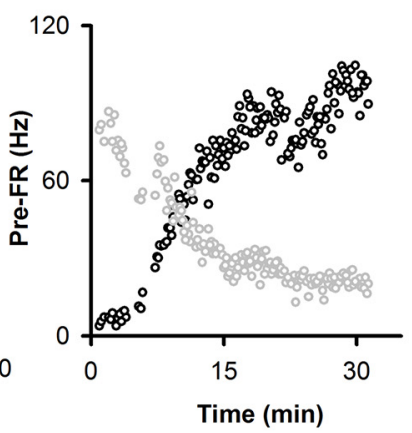

G
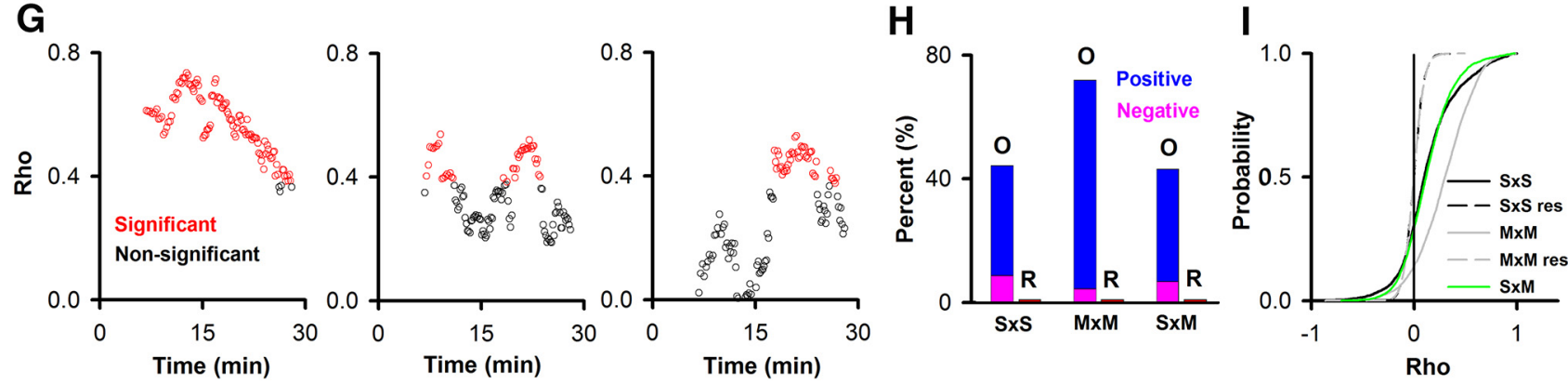

Figure 9. $M / T$ unit pairs show correlated changes in pre-FR for both SUs and MUs. $A-D$, Examples showing positive $(\boldsymbol{A}, \boldsymbol{B})$ or negative $(\boldsymbol{C}, \boldsymbol{D})$ correlation between SU pair pre-FRs. $A, C$, Dependence of the per-trial pre-FRs between these unit pairs. Both units pairs displayed correlated pre-FR ( $p$ value for the correlation coefficient, $\rho / p F D R=<0.001 /[0.02 \mathrm{SU} \times \mathrm{SU}, 0.02, \mathrm{SU} \times \mathrm{MU}, 0.04$ $\mathrm{MU} \times \mathrm{MU}], n=2972 \mathrm{SU} \times \mathrm{SU}, 4489 \mathrm{SU} \times \mathrm{MU}$, and $4719 \mathrm{MU} \times \mathrm{MU})$. Best-fit slope and intercept (Hz): $\boldsymbol{A}, 0.49,-0.1 ; \boldsymbol{C},-0.59,75 . \boldsymbol{B}, \boldsymbol{D}$, pre-FR as a function of time within the session for SUs in $\boldsymbol{A}$, $C . E, F$, Pseudocolor plot for $\rho$ and the significance $p$ value for $\rho$ determined for all unit pairs recorded in two different sessions. Gray represents nonsignificant $p$ values for $\rho(>\mathrm{pFDR}$ calculated for all windows within the session). G, Time course of the correlation coefficient, evaluated in a sliding window of 40 trials, for a subset of unit pairs for the session shown in $\boldsymbol{E}$. $\rho$ is significant for the points in red $(p<$ pFDR). $\boldsymbol{H}$, Percent of unit pairs that have significant correlation coefficients for pre-FRs. The number of unit pairs are 2972 (SU $\times$ SU), 4489 (SU $\times M U)$, and 4719 (MU $\times M U)$. Red represents a significant correlation. I, Cumulative probability histograms for the pre-FR correlation coefficients for all unit pairs showing more significant correlations in pre-FR between original versus resampled data. $S \times S, M \times S, M \times M$ : original data for $S U / M U$ unit pairs; $S \times S$ res, $M \times M$ Res: the pre-FR for unit 2 was calculated by random resampling the pre-FR histogram for 1 unit in the pair ( $M \times S$ Res, yields the same curve; data not shown). A Kolmogorov-Smirnov indicated that $S \times S / S \times M$ differ from $M \times M$ and $S \times S$ res $/ M \times M r e s . S \times M$ does not differ from $S \times S$, and $S \times S$ res does not differ from $M \times M$ res ( $p / p F D R:<0.05 / 0.05,15$ pairwise tests).

displayed a linear dependence for odor FR versus pre-FR and the percent of SU-odor pairs that displayed an odorant-induced change in FR dependent on pre-FR (a slope different from 1 in the odor FR vs pre-FR plot) were not significantly different between the passive and active tasks (Fig. $7 D, E, \chi^{2}, p$ value $>0.05$ in both cases). Furthermore, the distribution of slopes did not differ between the active and passive tasks (Fig. 7F, Kolmogorov-Smirnov, $p=0.62$, MWT, $p=0.43, n=43 \mathrm{SU}$-odor pairs for go-no go and 131 for go-go). Finally, we asked how the odorant-induced change in FR at low values of pre-FR differed between the two tasks. We focused on low pre-FR values because at those values we expect to find the largest increases in odor-induced FR due to the fact that the majority of the slopes are $<1$ (Fig. $5 A$ ). In addition, the odorantinduced responses are relevant because Kollo et al. (2014) postulated that "silent" $\mathrm{M} / \mathrm{T}$ cells dominate responses in the OB because they respond to odorants with large increases in FR. Because a minimum pre-FR cannot be defined in a finite data sample, we used 5th percentile pre-FR as a "low pre-FR value." When the change in FR at 5th percentile pre-FR was examined, we found a significant difference between the passive and active tasks (Fig. 7G, Kolmogorov-Smirnov, $p=0.0002$, MWT, $p=0.005, n=43$ SU-odor pairs for go-no go and 131 for go-go). These results indicate that pre-FR dependence of olfactory FR responses is reliant on behavioral status.

Trial-to-trial variability in licking accounts for a small fraction of the variance of the pre-FR

We then assessed whether the variability in pre-FR was accounted by trial-to-trial variability in licking during the prestimulus pe- 
A

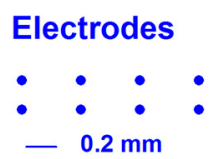

Tetrodes

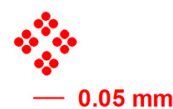

B

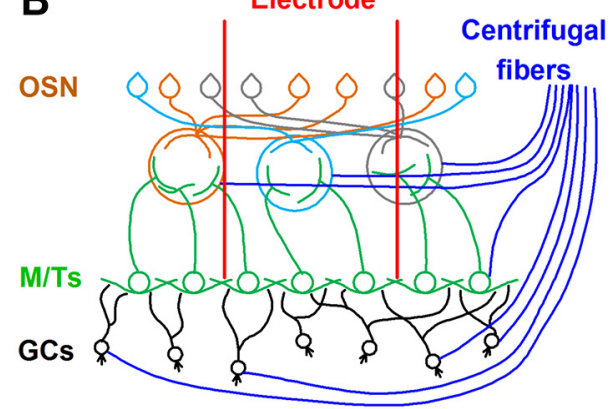

C

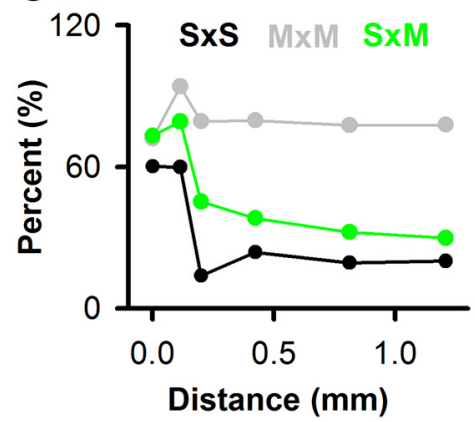

D

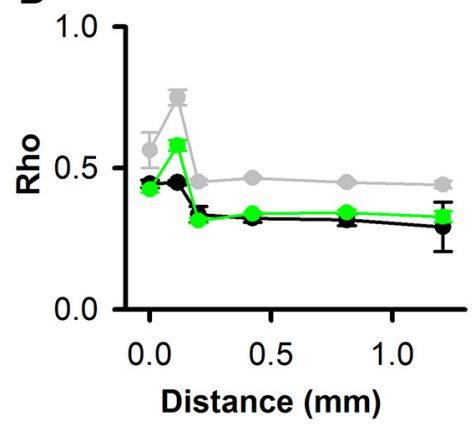

Figure 10. Mexican-hat dependence for unit pair pre-FR correlation dependence on the distance between electrodes/tetrodes. $\boldsymbol{A}$, Distances for multielectrode arrays (top, blue) (Doucette et al., 2011) or tetrodes (bottom, red) (Li et al., 2015). B, Simplified diagram of $O B$ circuitry illustrating the fact that the MTs spread in a cone under the glomerulus they innervate. GCs, Granule cells; OSN, olfactory sensory neuron. $C, \boldsymbol{D}$, Distance dependence of the percent of unit pairs with correlated pre-FRs ( $C$ ) and the correlation coefficient values $(\rho)$ for all unit pairs $(\boldsymbol{D}$, mean \pm SEM). The number of unit pairs is 12,180 ( $\rho, p$ value $<$ pFDR estimated at each distance bin).

riod. This analysis was performed for SUs recorded from both the go-no go and go-go behavioral sessions (Li et al., 2015). Figure $8 B 1, B 2$ shows the lick time course for a mouse engaged in the go-no go task whose behavioral performance is shown in Figure $8 A$ (red represents contact of the tongue with the lick tube). As the animal learns to differentiate between the odorants, the mice refrain from licking when exposed to the unrewarded odorant (Fig. 8B1). Figure $8 C$ shows an example of the dependence of pre-FR on the percent of the time the animal licks during the pre interval (percent lick). For this example, the data were not significantly correlated (correlation coefficient $=-0.18, p=0.024>$ $\mathrm{pFDR}=0.017$ for 383 SUs in the go-no go sessions).

When the statistical significance of the correlation between pre-FR and lick was determined for all SUs in the go-no go and go-go experiments, we found that approximately one-third of the SUs displayed significant correlation between pre-FR and percent lick (Fig. $8 D$, the pFDR for the 150 go-go SUs is 0.014 ). There was no difference between the two tasks for the percent of SUs that showed correlation between pre-FR and percent licks $\left(\chi^{2}\right.$, $p>0.05)$. The distribution of correlation coefficients is shown in Figure $8 F$. There was a statistical difference between go-no go and go-go for the cumulative distribution of the correlation coefficient (Fig. 8F, Kolmogorov-Smirnov, $p=0.04$ ), but there was no statistical difference for the mean correlation coefficient (Fig. $8 E$, unpaired $t$ test, $p=0.08$ ).

Finally, we estimated the percent of the variance for pre-FR that is accounted for by this dependence for SUs that displayed significant correlation between pre-FR and percent lick (129 SUs for go-no go and 43 SUs for go-go). The median of the variance explained by this dependence was $1.9 \%$ for go-no go and 1.3\% for go-go (Fig. $8 G$; these values differed statistically: MWT, $p=0.04$ ). Figure $8 H$ shows that the cumulative distributions for the variance accounted by the pre-FR versus percent lick dependence did not differ between the go-no go and go-go SUs (Kolmogorov-Smirnov, $p=0.15$ ). Thus, there is a dependence of pre FR on licking in one-third of the SUs, but this dependence accounts for a small percent of the pre-FR variance in most SUs.

\section{A substantial fraction of $\mathrm{M} / \mathrm{T}$ cell pairs display correlated trial-to-trial variation in pre-FR that is dependent on the distance between units}

In this study, we find odorant responses that display a linear dependence on pre-FR for a subset of M/T SUs. This raises the question whether the variability in pre-FR is due to behavioral factors, such as sniffing and licking, which would be expected to affect pre-FR uniformly in time and space as opposed to centrifugal feedback from other brain areas that could affect pre-FR differentially in different units. We determined whether pre-FR is correlated between unit pairs and whether this correlation varies uniformly for the different unit pairs as a function of time or as a function of distance between units in the SUs recorded in go-no go and go-go sessions (Doucette et al., 2011). Correlated firing is expected because measurements in anesthetized animals find correlated firing in 8\%-30\% of pairs of M/T cells (Kashiwadani et al., 1999; Egaña et al., 2005).

We assessed the correlation in FRs for unit pairs (both SUs and multiunits [MUs]) recorded from in all the go-no go and go-go sessions (Doucette et al., 2011; Li et al., 2015). We find that a substantial fraction of unit pairs display correlated pre-FR. Examples in Figure $9 A-D$ show pairs of simultaneously recorded units displaying positively or negatively correlated pre-FRs, and the pseudocolor plots in Figure 9E, $F$ show that a substantial fraction of simultaneously recorded unit pairs are significantly correlated in pre-FR with some M/T unit pairs displaying positively or negatively correlated trial-to-trial pre-FRs. In total, we evaluated trial-to-trial unit pair correlation in pre-FR in 12,180 unit pairs. A large percent of these pairs $(>40 \%)$ display variations in pre-FR that change throughout the session in a correlated manner (Fig. 9G,H). Correlated pre-FR was found in this fraction of unit pairs regardless of whether they were SUs or MUs. In all of these groups, the majority of the pairs displayed positive correlation coefficients significantly different from the coefficients calculated for pairs of units where the pre-FR for one of the units was resampled from its own pre-FR distribution (Fig. 9I, Kolmogorov-Smirnov, $p$ value/pFDR, $<0.05 / 0.05,15$ pairwise tests). Finally, when we quantified the unit pair correlation in pre-FR as a function of distance between recording sites, the dependence of the correlation coefficient and the percent of correlated unit pairs followed a Mexican hat dependence with significant correlations at distances as far as $1 \mathrm{~mm}$ (Fig. 10).

These findings suggest that neural circuits, perhaps via topdown modulation, mediate coordinated variation in pre-FR in ensembles of M/T cells. Interestingly, the time course of unit pair pre-FR correlation differed markedly between different units 


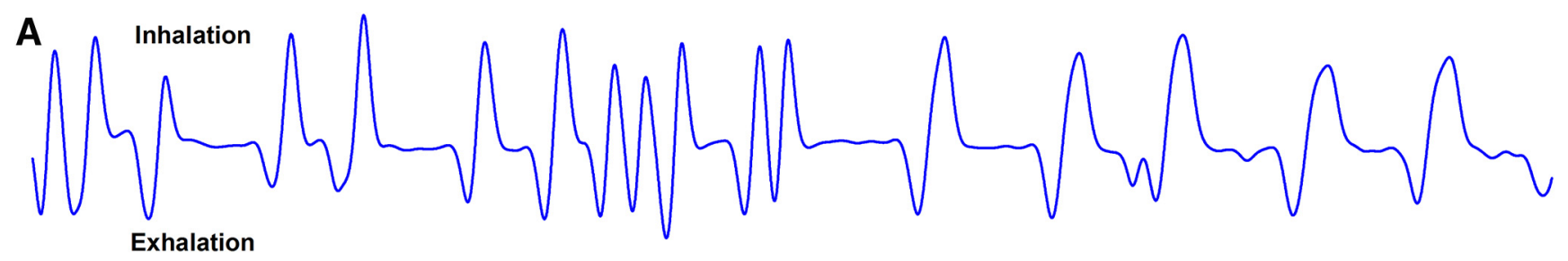

$2.5 \mathrm{~s}$

B

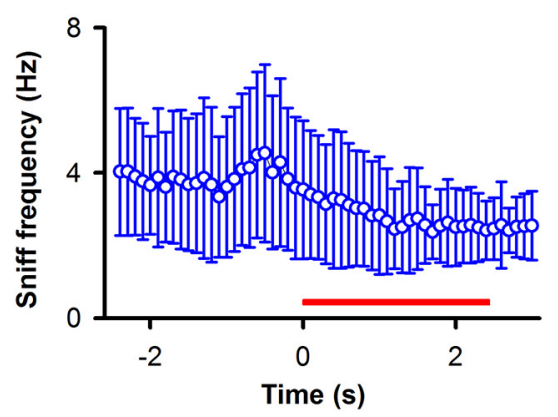

C

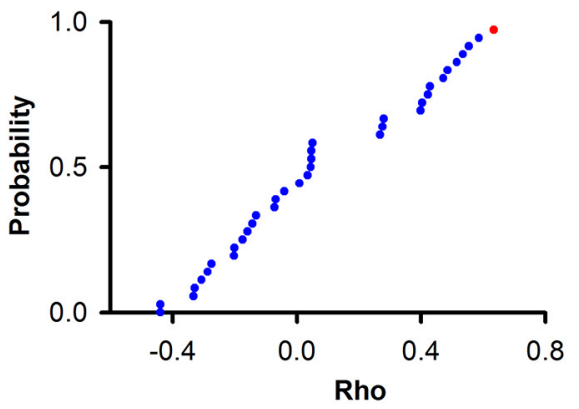

D

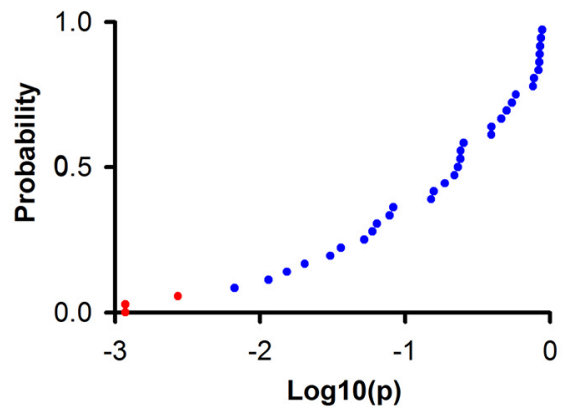

Figure 11. pre-FR is not correlated with the rate of sniff in the period before odorant addition. The period before odorant addition is defined as in Figure $4 A$. $A$, Example of the time course for nose pressure measured throughout a trial. Red line indicates application of odorant. $\boldsymbol{B}$, Time course for the mean sniff frequency \pm SD as a function of time (450 trials from 4 go-go sessions). C, $D$, Cumulative histogram for the correlation coefficient between per trial pre-FR and sniff frequency before odorant addition calculated for 36 odorant-unit pairs in 4 go-go sessions. $C$, Correlation coefficient $(\rho)$ for pre-FR and sniff frequency. $\boldsymbol{D}, p$ value for the correlation coefficient $(\rho)$. Blue represents not significant. Red represents significant $(p$ value/pFDR: $<0.003 / 0.003, n=36$ SUs).

within a session, as shown in Figure 9G. Thus, modulation of pre-FR correlation between different unit pairs is not exerted uniformly for all unit pairs, but rather, that pre-FR correlation is controlled independently and is specific for different sets of unit pairs.

\section{The pre-FR for the majority of units is not correlated with sniff rate}

We performed an analysis of the trial-to-trial dependence of pre-FR on the sniff frequency in 10 go-go sessions (Li et al., 2015). Figure $11 A$ shows the nasal pressure measured during a single trial, and Figure $11 B$ shows the mean sniff rate \pm SD as a function of time in the trial ( 450 trials from 10 sessions). In the go-go task, the sniff rate decreases after the animal enters the odor port. Figure $11 C, D$ shows, for the majority of SUs recorded in these sessions, that there is not a significant correlation between pre-FR and the mean sniff rate in the preodor period (red dots are significant, blue not significant; pFDR $<0.003, n=36$ SUs).

\section{Optogenetic stimulation of glomerular input, asynchronous with sniffing, yields changes in FR dependent on the pre-FR} We next explored whether light activation of channelrhodopsin 2 in axons of olfactory sensory neurons in the glomerular layer elicited FR changes in M/T SUs that were dependent on pre-FR using the SU data recorded by Li et al. (2014). As shown in Figure $12 \mathrm{~A}$, light stimulation was elicited, on purpose, at random phases within the sniff cycle ( $\mathrm{Li}$ et al., 2014). In this paradigm, the activation of glomerular input is not modulated by sniffing and takes place in the limited subset of axons illuminated by the $0.1 \mathrm{~mm}$ light fiber. Figure $12 B$ shows the activation time course, and Figure $12 C, D$ shows an example SU with a pre-FR-dependent response to light (light FR) (for an additional example unit, see Fig. 13A). Similar to the data presented in Figures 4 and 7, this unit displays a linear dependence of light FR on pre-FR with a slope different from 1. Further, this dependence has a slope that differs from 1, suggesting that for this unit light-evoked changes in FR depend on the pre-FR (Fig. 12D3). Overall, 44\% of the SU-light pairs (30 of 70) displayed a linear dependence of light FR on pre-FR (Fig. $12 G 1, p \leq \mathrm{pFDR}, \mathrm{pFDR}_{0 \text { degrees }}=0.0179, \mathrm{pFDR}_{-45 \text { degrees }}=0.0057$, $n=70$ SU-light pairs), and for $11 \%$ of SU-light pairs ( 8 of 70$)$ the slope of the best-fit line for light FR versus pre-FR differed from 1 (Fig. 12G2, $p \leq$ pFDR $=0.0057, n=70$ SU-light pairs). Together, these data demonstrate that the responses of a substantial number of $\mathrm{M} / \mathrm{T}$ units to olfactory sensory neuron input are dependent on their pre-FR and that this dependence is not affected by the phase of the animal's sniff cycle.

\section{Anesthesia affects the dependence of light-evoked responses on pre-FR}

As our data demonstrate that the dependence of odor FR on pre-FR changes with the behavioral status of the animal (Figs. 6, 7 ), we decided to test the hypothesis that the dependence of lightevoked FR on pre-FR changes when the animal is anesthetized. To achieve this, we evaluated responses of M/T SUs to light stimulation in mice anesthetized with $2 \%$ isoflurane in recordings performed immediately after the measurement in the awake animal. The recordings in the anesthetized mice were performed in the same animals whose optogenetic glomerular input activation was reported previously for the awake mouse (Li et al., 2014). Similar to awake mice, individual SUs demonstrated a dependence of light FR on pre-FR (for an example unit, see Fig. 13B). Overall, $54 \%$ of SUs (43 of 79) displayed a linear dependence of light FR on pre-FR (Fig. 12G1, $p \leq$ pFDR, pFDR degrees $=0.0077$, $\mathrm{pFDR}_{-45 \text { degrees }}=0.024, n=79$ SU-light pairs) and for $48 \%$ of SUs (38 of 79), this dependence had a slope that was different from 1 (Fig. 12G2, $p \leq$ pFDR $=0.0241, n=79$ SU-light pairs). Interestingly, despite not differing in pre-FR (Fig. 12E, MWT, $p=0.28, n=70 \mathrm{SU}$-light pairs in awake mice, $n=79 \mathrm{SU}$-light pairs in anesthetized mice), the slope of the best-fit line for light FR versus pre-FR was larger for SUs recorded in awake compared 
A-1 Sniff

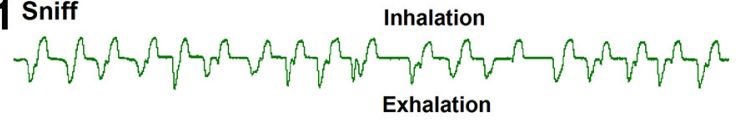

Sti

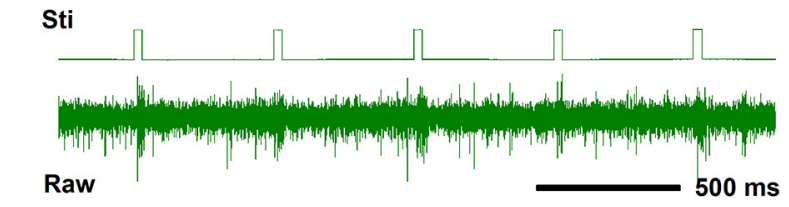

A-2
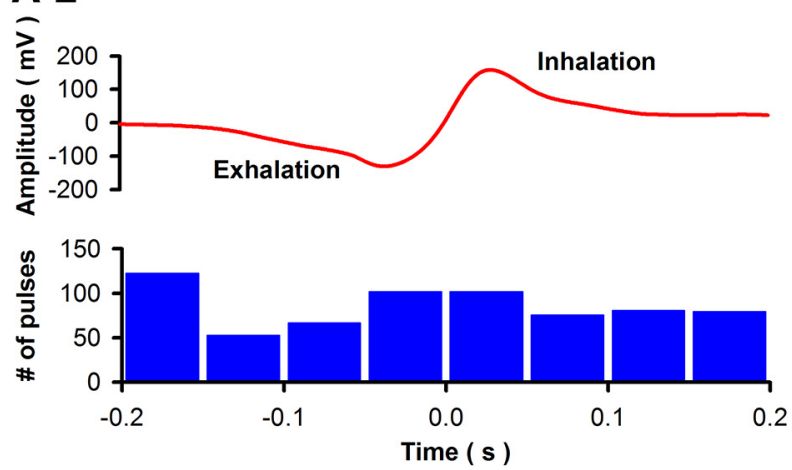

D-1

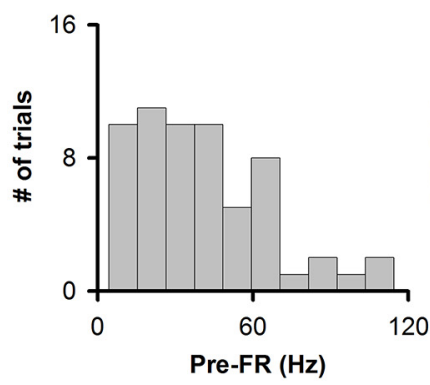

D-2

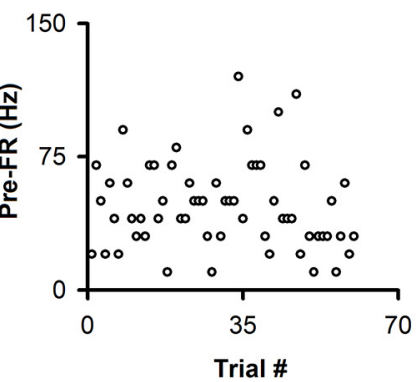

E

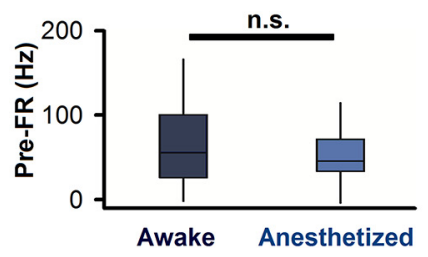

F

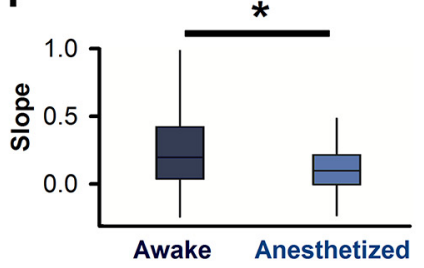

B

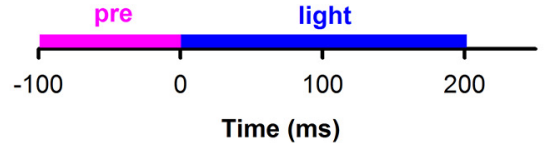

C-1

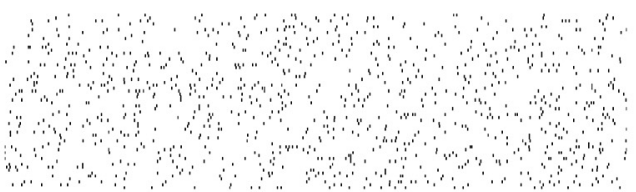

C-2
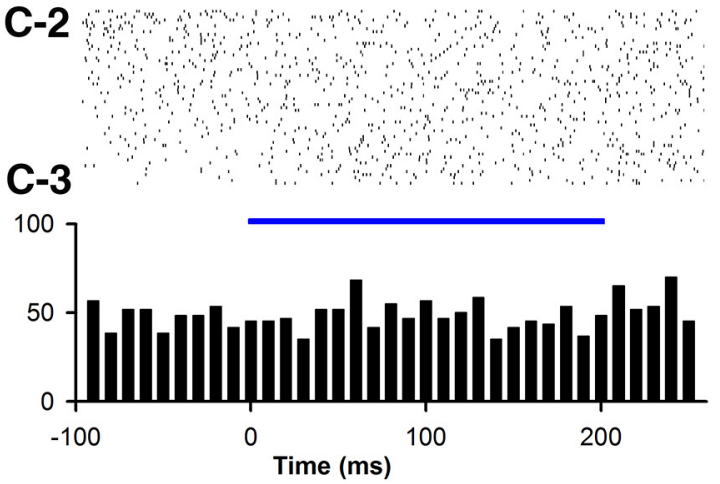

D-3

D-4
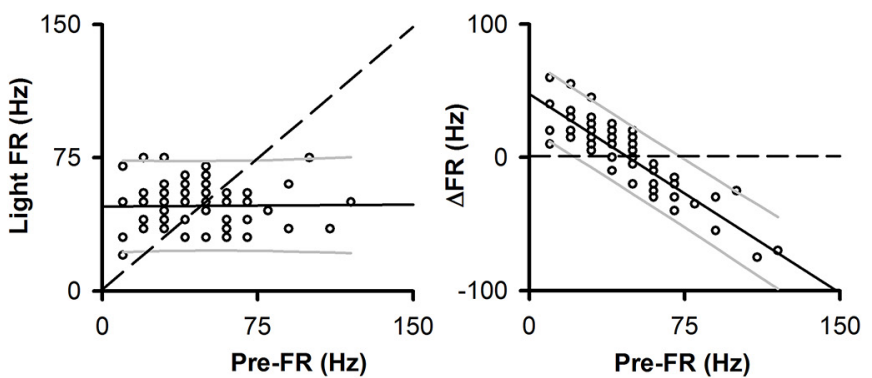

G-1

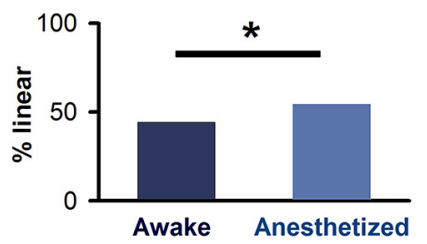

\section{G-2}

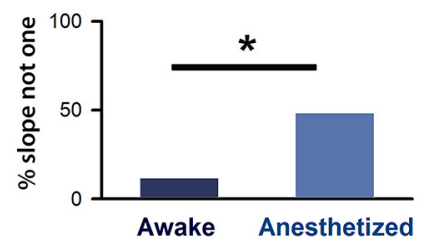

Figure 12. M/T cell responses to optogenetic activation of olfactory neuron axons, applied at random times, depend on pre-FR. $A, 0$ ptogenetic activation performed at random times within the sniff cycle. A1, Example of extracellular potential (Raw) and voltage recorded by the nose pressure sensor (Sniff). Light pulses (Sti). A2, Light pulses are not synchronized with sniff. Top, Average pressure voltage (681 sniffs). Bottom, Light activation. A Rayleigh test indicated that there was no specific distribution $(p<0.05)$ (reproduced from Li et al., 2014). B, Time course per trial. C, D, Example SU from awake mouse. C1, C2, Raster plots. C1, Unsorted. Bottom-top, First-last. C2, Sorted. Bottom-top, Lowest-highest pre-FR. C3, PSTH. D, Dependence of Light FR on pre-FR in an example SU (same SU as C). D1, Histogram of the per-trial pre-FRs. D2, Trial-to-trial variability in pre-FR. D3, Dependence of light FR on pre-FR. Dashed line indicates light $F R=$ pre-FR unity line. The slope of the best-fit line was linear and different from $1\left(p_{\text {linear }} \leq p F D R, p F D R_{0 \text { degrees }}=0.0179, p F D R=45\right.$ degrees $=0.0057, n=$ $70 \mathrm{SU}$-light pairs; $\mathrm{p}_{\text {different from } 1} \leq \mathrm{pFDR}=0.0057, n=70 \mathrm{SU}$-light pairs). Best-fit slope 0 , intercept $47.6 \mathrm{~Hz}$. D4, Dependence of $\Delta F R$ on pre-FR. Solid line indicates a best fit of the data. Dashed line indicates $\Delta F R=0$. Thin lines above and below the solid line indicate 5 th and 95 th percentile estimates. Best-fit slope and intercept (Hz): $-0.99,47.6$. $E$, Box-and-whiskers plot showing that mean pre-FR for SU-light pairs does not differ between awake $(n=70)$ and anesthetized $(n=79)$ mice (MWT, $p=0.2869)$. Conventions for the box-and-whisker plot: box represents the interquartile range (first to third quartile) and the median value (line), and whiskers extend to the most extreme data point within 1.5 times the interquartile range. $F$, Box-and-whiskers plot for slopes of best fit light FR versus pre-FR line for SU-light pairs differ between awake $(n=70)$ and anesthetized $(n=79)$ mice (MWT, $p<0.01)$. G1, Percent of SU-light pairs with a linear dependence of light FR on pre-FR differs between awake and anesthetized mice $\left(\chi^{2}, p<0.05\right)$. G2, Percent of SU-light pairs with a linear dependence with a slope distinct from 1 differs between awake and anesthetized mice $\left(\chi^{2}, p<0.0001\right)$. ${ }^{*}$ Statistically significant.

with anesthetized mice (Fig. 12F: MWT, $p<0.01$; Fig. 13C: Kolmogorov-Smirnov, $p<0.05 ; n=70$ SU-light pairs in awake mice, $n=79 \mathrm{SU}$-light pairs in anesthetized mice). Further, the percent of units with a linear dependence of light FR on pre-FR and with a slope distinct from 1 was greater in the anesthetized mice compared with the awake mice (Fig. $12 G, G 1: \chi^{2}, p$ value $<$
$0.05, G 2: \chi^{2}, p$ value $\left.<0.0001\right)$. These data provide additional evidence consistent with the notion that the dependence of $\mathrm{M} / \mathrm{T}$ cell firing on pre-FR differs across behavioral states. In particular, the differences in the slope of the light FR-pre-FR dependence between awake and anesthetized mice (Figs. 12F, Fig. 13C) suggest that, on any given trial, an individual M/T unit will be more 

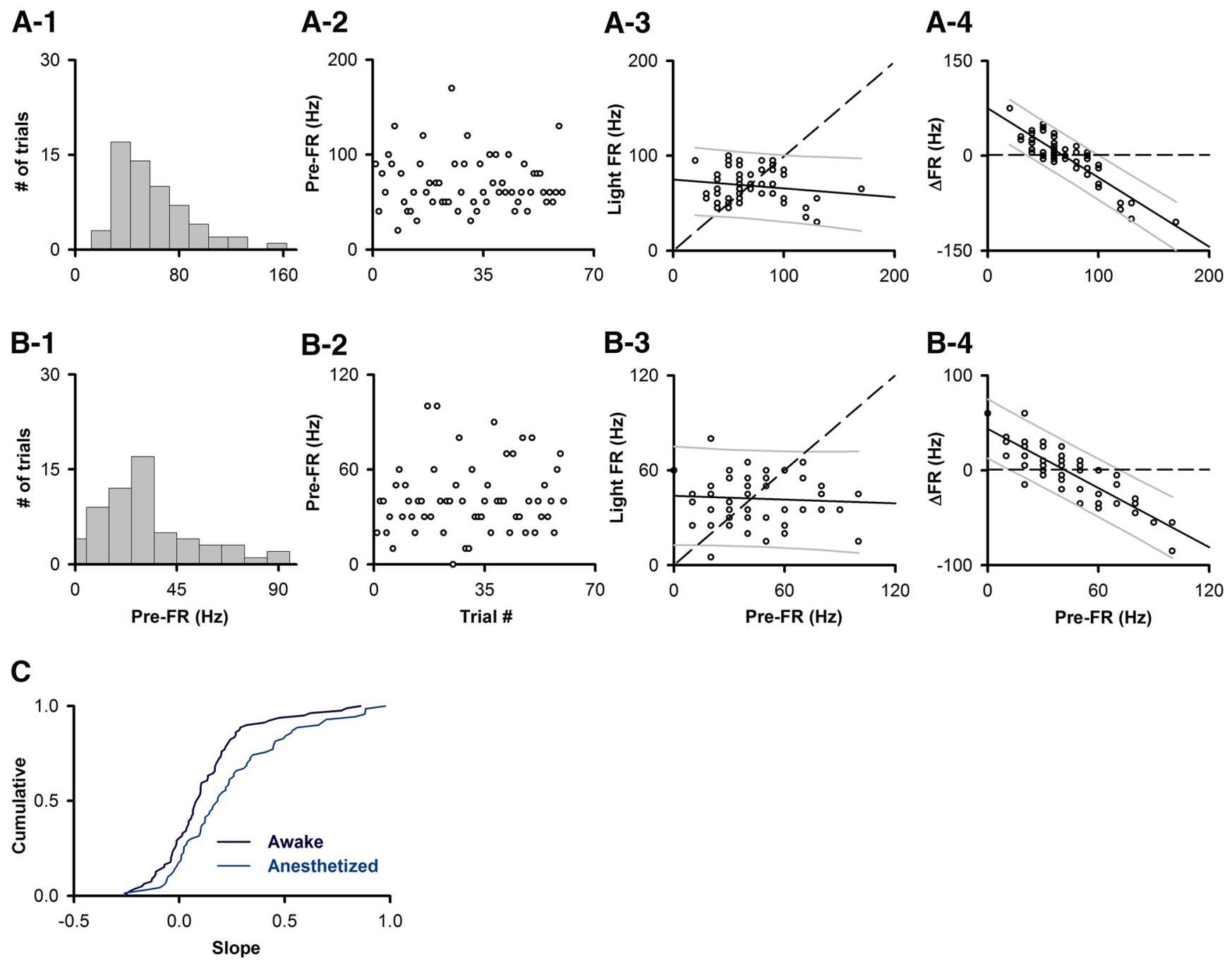

Figure 13. M/T cell responses to optogenetic activation of olfactory neuron axons, applied at random times, depend on pre-FR. $\boldsymbol{A}, \boldsymbol{B}$, Dependence of light $F R$ on pre-FR in two example SUs. $\boldsymbol{A}$, Recorded from an SU in an awake animal. $\boldsymbol{B}$, Recorded in an anesthetized animal. $\boldsymbol{A}$, $\boldsymbol{B} \mathbf{1}$, Histogram of the per-trial pre-FRs. $\mathbf{A 2}, \mathbf{B 2}$, Trial-to-trial variability in pre-FR. $\boldsymbol{A}$, $\boldsymbol{B} \mathbf{3}$, Dependence of light FR on pre-FR. Dashed line indicates light $F R=$ pre-FR unity line. The slope of the best-fit line was linear and different than 1 ( $A 3: p_{\text {linear }} \leq p F D R, p F D R_{0 \text { degrees }}=0.0179$, pFDR -45 degrees $=0.0057$,

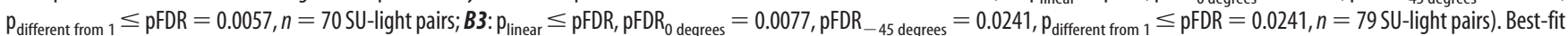
slope and intercept (Hz): $A 3,-0.09,74.9$. B3, $-0.04,43.9 . A 4, B 4$, Dependence of $\Delta F R$ on pre-FR. Solid line indicates a best fit of the data. Dashed line indicates $\Delta F R=0$. Thin lines above and below the solid line indicate 5 th and 95 th percentile estimates. Best-fit slope and intercept (Hz): $A 4,-1.09,74.9 . B 4,-1.04,43.9$. C, Cumulative probabilities for the slope of light FR versus pre-FR best-fit line for SUs recorded in awake and anesthetized animals. The distributions of the slopes differed between groups (Kolmogorov-Smirnov, $p<0.05, n=70$ awake SUs, $n=79$ anesthetized SUS).

likely to show a change in FR in response to input from olfactory sensory neurons in anesthetized compared with awake mice.

\section{Discussion}

The odorant-induced change in FR of M/T cells depends on the pre-FR

In his insightful 1950 manuscript (Adrian, 1950), Lord Adrian found a significant decrease in odorant-induced changes in FR that appeared to depend on an increase in the pre-FR as the animal awoke from anesthesia. He ended this paper with an interesting speculation: "In particular the failure of olfactory sensation after repeated stimuli can be compared with our inability to pay continued attention to uninteresting sounds." Here we explored whether the differences in odorant responses of $\mathrm{M} / \mathrm{T}$ cells from trial-to-trial in an awake behaving animal were due to the dependence of odorant responses on pre-FR. We find that, in a substantial fraction of M/T units, the change in FR elicited by the odor depends on pre-FR (Figs. 4, 5) and that this dependence changes when both odorants are rewarded equally (Fig. 7), when the animal is anesthetized (Figs. 12,13), and in trials when the animal makes a mistake while learning to refrain from responding to the unrewarded odorant (Fig. 6).

The dependence of the change in FR elicited by the odor on pre-FR is likely due to a set point for the FR of the unit in the presence of a particular odor. Thus, Figure 4D4 shows an odorant-induced increase in pre-FR that appears to reach a maximum set point. Thus, in this case the odorant-induced change in FR is always positive and decreases as pre-FR increases. This responsiveness is expected for a neuron within a neuronal circuit that stimulates it to its maximum FR. On the other hand, Figures $4 E 4$ and $7 B 3$ show units that respond to the odors with a decrease in FR. That is expected from neurons influenced by an inhibitory circuit that decreases its activity to a minimum set point FR. Regardless, the change in FR elicited by the odorant that will be detected by downstream neurons depends on pre-FR, and this 
dependence is modified by the behavioral status of the animal. In addition, the pre-FR of different units is correlated and highly variable on a trial-per-trial basis (Fig. 9).

\section{The dependence of odorant-induced changes in FR on the pre-FR differs between different behavioral states}

Studies subsequent to Lord Adrian's generally find robust responses in anesthetized animals, and higher pre-FR for M/T cells with odorant-induced changes in FR that vary from robust to sparse virtually absent in awake animals, even when the response is aligned sniffs (Kay and Laurent, 1999; Rinberg et al., 2006; Davison and Katz, 2007; Fuentes et al., 2008; Cury and Uchida, 2010; Doucette et al., 2011; Shusterman et al., 2011; Gschwend et al., 2012; Kato et al., 2012; Blauvelt et al., 2013; Fukunaga et al., 2014). We find that the dependence of light FR on pre-FR persists in the anesthetized mouse. The percent of slopes for this dependence distinct from one was greater in the anesthetized mice (Fig. 12G2). This is likely due to the fact that $\mathrm{M} / \mathrm{T}$ cells are more responsive in the anesthetized state.

Although information on odor identity is likely conveyed by spike firing within the sniff cycle (Cury and Uchida, 2010; Shusterman et al., 2011; Gschwend et al., 2012; Li et al., 2015), the diversity of observations of odorant-induced responses in the awake animals raise the question whether these responses do differ depending on the behavioral status of the animal in different laboratories. Interestingly, there is large inherent variation in pre-FR and odorant responsiveness in M/T cells. Indeed, a recent study in the awake mouse found that a subset of M/T cells with low pre-FR responded to odor with a substantial increase in FR, and these investigators raised the question whether these "silent" $\mathrm{M} / \mathrm{T}$ cells dominate odor responses in the $\mathrm{OB}$ (Kollo et al., 2014). Our findings suggest that M/T cells should not classified as "silent" versus intrinsically active. Rather, our study indicates that the intrinsic activity of $\mathrm{M} / \mathrm{T}$ cells varies on a trial-by-trial basis depending on the behavioral status and that in each trial different subsets of "silent" cells dominate the response. This is consistent with findings in a subset of the awake behaving animal studies showing that the responsiveness to odorants differed depending on the behavioral status of the animal (Fuentes et al., 2008; Doucette et al., 2011; Kato et al., 2012).

We find that the odorant-induced response of M/T cells is negatively correlated with the pre-FR on a trial-to-trial basis. Importantly, optogenetic stimulation of olfactory sensory neuron axons showed that the dependence of activation of M/T cells on pre-FR was sniff phase-independent (Figs. 11, 12) and variation in licks only accounted for a small percent of the pre-FR variance in one-third of the units (Fig. 8). In addition, trial-totrial variation of pre-FR occurred in ensembles of $\mathrm{M} / \mathrm{T}$ cells with correlated pre-FR suggesting common regulation by a local circuit (Kay and Freeman, 1998). The variation in pre-FR correlation during a session was heterogeneous in different unit pairs indicating that this circuit does not affect the entire M/T cell ensemble as it would happen for homogeneous modulation by processes, such as adrenergic regulation (Bouret and Sara, 2004, 2005) and regulation of neuronal activity by sniff (Cury and Uchida, 2010; Shusterman et al., 2011; Wachowiak, 2011; Li et al., 2015). In addition, the $\mathrm{M} / \mathrm{T}$ unit pair correlation in pre-FR occurred in cells spaced up to $1 \mathrm{~mm}$ (Fig. 10), consistent with observations of long-range synchronous firing in these OB relay neurons (Doucette et al., 2011). This local circuit mechanism that modulates subsets of M/T cells heterogeneously and mediates pre-FR correlation at long distance, could be, for example, the dopaminergic periglomerular cells that mediate intraglomerular interactions (Kiyokage et al., 2010; Banerjee et al., 2015).

\section{Modulation of M/T cell pre-FR may allow judicious subsampling of the multidimensional spatiotemporal olfactory input space}

Interestingly, preafferent anticipatory events were proposed in the OB based on field potential measurements (Kay and Freeman, 1998). Consistent with this proposal, we find that pre-FR activity is affected by the behavioral status with larger pre-FR values in the passive task compared with the active task where odorant valence plays a role in decision making. In addition, we show that the pre-FR is different when an animal makes a mistake in the active associative learning task suggesting that pre-FR reflects an anticipatory cue (Gardner and Fontanini, 2014). These changes in pre-FR are likely mediated by centrifugal input, such as piriform cortex or prestimulus forebrain input to the $\mathrm{OB}$ (Kay and Freeman, 1998; Zelano et al., 2005; Buonomano and Maass, 2009; Gire et al., 2013; Nunez-Parra et al., 2013; Linster and Fontanini, 2014; Bota et al., 2015; Hangya et al., 2015; Otazu et al., 2015; Devore et al., 2016). Our findings show, for the first time, that the responsiveness of a second-order cell in the olfactory system, that receives massive parallel input from $>1200$ olfactory receptors (Buck and Axel, 1991; Touhara and Vosshall, 2009), depends on the pre-FR and that this dependence is modified by behavioral status of the animal. This is consistent with findings of UP and DOWN states in mitral cells found in slice recordings, postulated to affect olfactory information processing (Carlson et al., 2000; Roux et al., 2015), and with the dependence of responses on baseline neural activity in sensory cortex and other model organisms (Buonomano and Maass, 2009; Nishida et al., 2014; Zhang et al., 2014; Gordus et al., 2015). This dependence has a consequence on neuronal responses in the presence of high variability of pre-FR in the awake animal. The fact that pre-FR dependence of the stimulus response is modified in different behavioral states could reflect modulation by processes, such as changes in attention, motivation, or other preafferent anticipatory events (Devore et al., 2016). Perhaps the brain deals with this complex computation by engaging ensembles of $\mathrm{M} / \mathrm{T}$ cells depending on the context as a performer does playing the piano and evaluating stimulus-induced changes in FR using an algorithm analogous to Extreme Value Theory (de Haan and Ferreira, 2006).

\section{References}

Adrian ED (1950) The electrical activity of the mammalian olfactory bulb. Electroencephalogr Clin Neurophysiol 2:377-388.

Banerjee A, Marbach F, Anselmi F, Koh MS, Davis MB, Garcia da Silva P, Delevich K, Oyibo HK, Gupta P, Li B, Albeanu DF (2015) An interglomerular circuit gates glomerular output and implements gain control in the mouse olfactory bulb. Neuron 87:193-207.

Benjamini Y, Yekutieli D (2001) The control of the false discovery rate in multiple testing under dependency. Ann Stat 29:1165-1188.

Benjamini Y, Yekutieli D (2005) Quantitative trait loci analysis using the false discovery rate. Genetics 171:783-790.

Blauvelt DG, Sato TF, Wienisch M, Knöpfel T, Murthy VN (2013) Distinct spatiotemporal activity in principal neurons of the mouse olfactory bulb in anesthetized and awake states. Front Neural Circuits 7:46.

Bota M, Sporns O, Swanson LW (2015) Architecture of the cerebral cortical association connectome underlying cognition. Proc Natl Acad Sci U S A 112:E2093-E2101.

Bouret S, Sara SJ (2004) Reward expectation, orientation of attention and locus coeruleus-medial frontal cortex interplay during learning. Eur J Neurosci 20:791-802.

Bouret S, Sara SJ (2005) Network reset: a simplified overarching theory of locus coeruleus noradrenaline function. Trends Neurosci 28:574-582. 
Buck L, Axel R (1991) A novel multigene family may encode odorant receptors: a molecular basis for odor recognition. Cell 65:175-187.

Buonomano DV, Maass W (2009) State-dependent computations: spatiotemporal processing in cortical networks. Nat Rev Neurosci 10:113-125.

Carlson GC, Shipley MT, Keller A (2000) Long-lasting depolarizations in mitral cells of the rat olfactory bulb. J Neurosci 20:2011-2021.

Curran-Everett D (2000) Multiple comparisons: philosophies and illustrations. Am J Physiol Regul Integr Comp Physiol 279:R1-R8.

Cury KM, Uchida N (2010) Robust odor coding via inhalation-coupled transient activity in the mammalian olfactory bulb. Neuron 68:570-585.

Davison IG, Katz LC (2007) Sparse and selective odor coding by mitral/ tufted neurons in the main olfactory bulb. J Neurosci 27:2091-2101.

de Haan L, Ferreira A (2006) Extreme value theory. an introduction. New York: Springer.

Devore S, Pender-Morris N, Dean O, Smith D, Linster C (2016) Basal forebrain dynamics during non-associative and associative olfactory learning. J Neurophysiol 115:423-433.

Doucette W, Restrepo D (2008) Profound context-dependent plasticity of mitral cell responses in olfactory bulb. PLoS Biol 6:e258.

Doucette W, Gire DH, Whitesell J, Carmean V, Lucero MT, Restrepo D (2011) Associative cortex features in the first olfactory brain relay station. Neuron 69:1176-1187.

Eeckman FH, Freeman WJ (1991) Asymmetric sigmoid non-linearity in the rat olfactory system. Brain Res 557:13-21.

Egaña JI, Aylwin ML, Maldonado PE (2005) Odor response properties of neighboring mitral/tufted cells in the rat olfactory bulb. Neuroscience 134:1069-1080.

Fontanini A, Katz DB (2008) Behavioral states, network states, and sensory response variability. J Neurophysiol 100:1160-1168.

Fuentes RA, Aguilar MI, Aylwin ML, Maldonado PE (2008) Neuronal activity of mitral-tufted cells in awake rats during passive and active odorant stimulation. J Neurophysiol 100:422-430.

Fukunaga I, Herb JT, Kollo M, Boyden ES, Schaefer AT (2014) Independent control of gamma and theta activity by distinct interneuron networks in the olfactory bulb. Nat Neurosci 17:1208-1216.

Gardner MP, Fontanini A (2014) Encoding and tracking of outcomespecific expectancy in the gustatory cortex of alert rats. J Neurosci 34:13000-13017.

Gire DH, Restrepo D, Sejnowski TJ, Greer C, De Carlos JA, LopezMascaraque L (2013) Temporal processing in the olfactory system: can we see a smell? Neuron 78:416-432.

Gordus A, Pokala N, Levy S, Flavell SW, Bargmann CI (2015) Feedback from network States generates variability in a probabilistic olfactory circuit. Cell 161:215-227.

Gray CM, Maldonado PE, Wilson M, McNaughton B (1995) Tetrodes markedly improve the reliability and yield of multiple single-unit isolation from multi-unit recordings in cat striate cortex. J Neurosci Methods 63:43-54.

Gschwend O, Beroud J, Carleton A (2012) Encoding odorant identity by spiking packets of rate-invariant neurons in awake mice. PLoS One 7:e30155.

Gschwend O, Abraham NM, Lagier S, Begnaud F, Rodriguez I, Carleton A (2015) Neuronal pattern separation in the olfactory bulb improves odor discrimination learning. Nat Neurosci 18:1474-1482.

Hangya B, Ranade SP, Lorenc M, Kepecs A (2015) Central cholinergic neurons are rapidly recruited by reinforcement feedback. Cell 162:1155-1168.

Heyward P, Ennis M, Keller A, Shipley MT (2001) Membrane bistability in olfactory bulb mitral cells. J Neurosci 21:5311-5320.
Jeanne JM, Sharpee TO, Gentner TQ (2013) Associative learning enhances population coding by inverting interneuronal correlation patterns. Neuron 78:352-363.

Kashiwadani H, Sasaki YF, Uchida N, Mori K (1999) Synchronized oscillatory discharges of mitral/tufted cells with different molecular receptive ranges in the rabbit olfactory bulb. J Neurophysiol 82:1786-1792.

Kato HK, Chu MW, Isaacson JS, Komiyama T (2012) Dynamic sensory representations in the olfactory bulb: modulation by wakefulness and experience. Neuron 76:962-975.

Kay LM, Freeman WJ (1998) Bidirectional processing in the olfactorylimbic axis during olfactory behavior. Behav Neurosci 112:541-553.

Kay LM, Laurent G (1999) Odor- and context-dependent modulation of mitral cell activity in behaving rats. Nat Neurosci 2:1003-1009.

Kiyokage E, Pan YZ, Shao Z, Kobayashi K, Szabo G, Yanagawa Y, Obata K, Okano H, Toida K, Puche AC, Shipley MT (2010) Molecular identity of periglomerular and short axon cells. J Neurosci 30:1185-1196.

Kollo M, Schmaltz A, Abdelhamid M, Fukunaga I, Schaefer AT (2014) 'Silent' mitral cells dominate odor responses in the olfactory bulb of awake mice. Nat Neurosci 17:1313-1315.

Li A, Gire DH, Bozza T, Restrepo D (2014) Precise detection of direct glomerular input duration by the olfactory bulb. J Neurosci 34:16058-16064.

Li A, Gire DH, Restrepo D (2015) Y spike-field coherence in a population of olfactory bulb neurons differentiates between odors irrespective of associated outcome. J Neurosci 35:5808-5822.

Linster C, Fontanini A (2014) Functional neuromodulation of chemosensation in vertebrates. Curr Opin Neurobiol 29:82-87.

Nishida S, Tanaka T, Shibata T, Ikeda K, Aso T, Ogawa T (2014) Dischargerate persistence of baseline activity during fixation reflects maintenance of memory-period activity in the macaque posterior parietal cortex. Cereb Cortex 24:1671-1685.

Nunez-Parra A, Maurer RK, Krahe K, Smith RS, Araneda RC (2013) Disruption of centrifugal inhibition to olfactory bulb granule cells impairs olfactory discrimination. Proc Natl Acad Sci U S A 110:14777-14782.

Otazu GH, Chae H, Davis MB, Albeanu DF (2015) Cortical feedback decorrelates olfactory bulb output in awake mice. Neuron 86:1461-1477.

Poulet JF, Petersen CC (2008) Internal brain state regulates membrane potential synchrony in barrel cortex of behaving mice. Nature 454:881-885.

Rinberg D, Koulakov A, Gelperin A (2006) Sparse odor coding in awake behaving mice. J Neurosci 26:8857-8865.

Roux L, Madar A, Lacroix MM, Yi C, Benchenane K, Giaume C (2015) Astroglial connexin 43 hemichannels modulate olfactory bulb slow oscillations. J Neurosci 35:15339-15352.

Shusterman R, Smear MC, Koulakov AA, Rinberg D (2011) Precise olfactory responses tile the sniff cycle. Nat Neurosci 14:1039-1044.

Stubblefield EA, Thompson JA, Felsen G (2015) Optogenetic cholinergic modulation of the mouse superior colliculus in vivo. J Neurophysiol 114:978-988.

Touhara K, Vosshall LB (2009) Sensing odorants and pheromones with chemosensory receptors. Annu Rev Physiol 71:307-332.

Wachowiak M (2011) All in a sniff: olfaction as a model for active sensing. Neuron 71:962-973.

Zelano C, Bensafi M, Porter J, Mainland J, Johnson B, Bremner E, Telles C, Khan R, Sobel N (2005) Attentional modulation in human primary olfactory cortex. Nat Neurosci 8:114-120.

Zhang M, Wang X, Goldberg ME (2014) A spatially nonselective baseline signal in parietal cortex reflects the probability of a monkey's success on the current trial. Proc Natl Acad Sci U S A 111:8967-8972. 